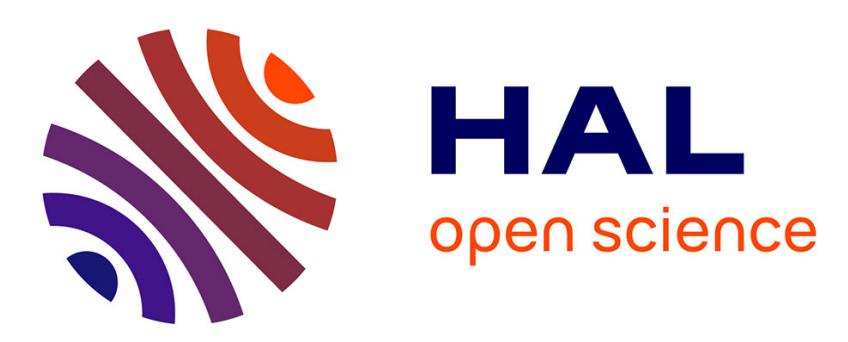

\title{
Atypical secondary electron emission yield curves of very thin SiO2 layers: Experiments and modeling
}

Charles Rigoudy, Kremena Makasheva, M. Belhaj, Sarah Dadouch, G. Teyssedre, Laurent Boudou

\section{- To cite this version:}

Charles Rigoudy, Kremena Makasheva, M. Belhaj, Sarah Dadouch, G. Teyssedre, et al.. Atypical secondary electron emission yield curves of very thin SiO2 layers: Experiments and modeling. Journal of Applied Physics, 2021, 130 (13), pp.135305. 10.1063/5.0056218 . hal-03371196

\section{HAL Id: hal-03371196 https://hal.science/hal-03371196}

Submitted on 8 Oct 2021

HAL is a multi-disciplinary open access archive for the deposit and dissemination of scientific research documents, whether they are published or not. The documents may come from teaching and research institutions in France or abroad, or from public or private research centers.
L'archive ouverte pluridisciplinaire HAL, est destinée au dépôt et à la diffusion de documents scientifiques de niveau recherche, publiés ou non, émanant des établissements d'enseignement et de recherche français ou étrangers, des laboratoires publics ou privés. 


\title{
Atypical secondary electron emission yield curves of very thin $\mathrm{SiO}_{2}$ layers: experiments and modeling
}

\author{
C. Rigoudy, ${ }^{1,2}$ K. Makasheva, ${ }^{1}$ M. Belhaj, ${ }^{2}$ S. Dadouch, ${ }^{2}$ G. Teyssedre, ${ }^{1}$ and L. Boudou ${ }^{1}$ \\ ${ }^{1}$ LAPLACE (Laboratoire Plasma et Conversion d'Energie), Université de Toulouse; CNRS UPS, INPT; \\ 118 route de Narbonne, F-31062 Toulouse cedex 9, France \\ ${ }^{2}$ ONERA -The French Aerospace Laboratory, FR-31062 Toulouse cedex 4, France
}

Corresponding author: laurent.boudou@laplace.univ-tlse.fr

Abstract - The secondary electron emission phenomenon often refers to the emission of electrons as a result of the interaction of impinging energetic electrons with the surface of a material. Although it is fairly well described for metals, with a typical shape of the total electron emission yield (TEEY) first increasing to reach a maximum and then decreasing along with the energy increase of the primary electrons, there is still a lack of data and detailed analysis for dielectrics, in particular thin layers. The present work proposes a new insight in the electron emission phenomenon from very thin dielectric layers. It reports on the TEEY from very thin $\mathrm{SiO}_{2}$ layers, less than $100 \mathrm{~nm}$. It is found that a departure from the typical shape of the TEEY curve occurs for primary electrons with energy of around $1 \mathrm{keV}$. The TEEY curve presents a dip, a local minimum that might be as deep as below 1. This atypical shape depends substantially on the layer thickness. The measured TEEY are compared to an electron emission 1D-model in which we consider the combined effect of the space-charge electric field induced by trapped charges in the dielectric layer and of the processes of field dependent conductivity (FDC) and radiation induced conductivity (RIC) on the fate of secondary electrons. Those mechanisms govern the charge transport in the dielectric, and consequently the electron emission. The effects of the $\mathrm{SiO}_{2}$ layer thickness, incidence angle of the primary electrons and an applied external electric field on the TEEY curves are reported. 


\section{INTRODUCTION}

Electron emission is a process of release of electrons from materials. A way to achieve it is via irradiation of the material by energetic species. Thus the process results from the energy transfer of the impinging energetic species (electrons, ions, atoms, photons) to the material surface. The electron emission is a complex phenomenon that depends on many parameters related to the primary particles (nature, energy, incident angle distribution, etc.) and to the studied material (nature, conductivity, surface state - morphology/roughness/contamination, etc. ${ }^{1-3}$ This physical phenomenon is largely involved in scanning electron microscopy $(\mathrm{SEM}),{ }^{4-6}$ plasma physics,${ }^{7}$ space applications,${ }^{8,9}$ particle accelerators,${ }^{10}$ among other fields, and lays down the principle of operation of many devices. ${ }^{11-14}$ Given the large number of applications using electron emission for their operation, a lot of effort has been made during the last century to determine the electron emission yield from different materials, being conducting, semiconducting or insulating ones. ${ }^{1,2}$ However, due to some peculiarities related to the nature of materials, the electron emission phenomenon from thin dielectric layers remains an open field of research.

Without loss of generality, the analysis here is limited to electron irradiation. Under electron irradiation, the total electron emission yield (TEEY), encompasses backscattered electrons and secondary electron emissions, referred as BEEY and SEEY, respectively. The TEEY is defined as the ratio of total number of emitted electrons to the number of primary (incident) electrons. Depending on the energy of incident electrons, as well as the surface composition, morphology and physical properties of the material, the current emitted from the material can be larger or smaller than the incident beam current. Besides, due to the dielectric charging phenomenon, a particular attention is required when characterizing insulating materials. If neutrality cannot be restored instantaneously, as for the case of metals, the dielectric material gets either positively or negatively charged. In this way, the electron irradiation induces potential gradients that can reach thousands of Volts between the piece parts and trigger electrical discharges, with the latter leading to a damage of the device. Such process can entail important failures in space applications, for example. ${ }^{15,16}$ Thus, the dielectric charging phenomenon has several consequences on the TEEY: $(i)$ it modifies the energy of primary electrons, hence their penetration depth; (ii) it impacts the transport of secondary electrons in the dielectric and (iii) it alters their release to the vacuum. These features of dielectric materials make the experimental determination of the true TEEY (the one corresponding to an uncharged dielectric surface) delicate, and the modeling of TEEY difficult. For illustration, the TEEY curve as a function of the energy of primary electrons is shown in 
Fig. 1. The typical TEEY curve, largely reported in the literature, is characterized by a maximum of the TEEY (at energy $\mathrm{E}_{\max }$ ) and two specific values called crossover energies (noted $\mathrm{E}_{\mathrm{C} 1}$ and $\mathrm{E}_{\mathrm{C} 2}$ ) for which the TEEY = 1, i.e., the number of emitted electrons equals the number of incident electrons. But for thin $\mathrm{SiO}_{2}$ layers (100 nm and less) the TEEY curve does not always follow this typical behavior. ${ }^{17-19}$ Indeed, the TEEY curve exhibits a dip in the emission yield for energies between 500 and $1000 \mathrm{eV}$, which will be referred hereafter to as local minimum, and noted $Y_{\min }$. The red curve in Fig. 1 shows the shape of such atypical TEEY curve. There exists a few reports in the literature about such anomaly of the TEEY curve of thin $\mathrm{SiO}_{2}$ layers ${ }^{17,18}$ and spacecraft insulating materials ${ }^{19}$ but the origin of this phenomenon has not been completely revealed. In some cases, it can be attributed to dielectric charging of the studied dielectric layers, as a consequence of continuous electron irradiation of the sample in the experiment. A competition between the penetration depth of primary electrons and the thickness of dielectric layer, again in relation with the dielectric charging phenomenon, could also be surmised as a factor leading to the appearance of a local minimum in the TEEY curve. ${ }^{17-19}$

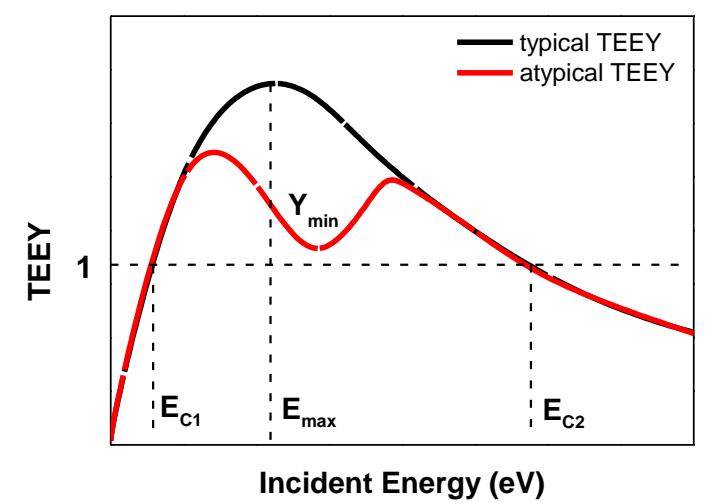

FIG. 1. Schematic representation of a typical shape of the TEEY curve (black curve) and an atypical shape of TEEY curve observed for thin dielectric layers (red curve).

Thus, the objective of this work is to shed light on the origin of the atypical TEEY curve of thin dielectric layers when irradiated by electrons. Our approach relies on combined experimental and theoretical (modeling) study of thin $\mathrm{SiO}_{2}$ layers. In the experiments, the beam of primary electrons is pulsed in order to limit the dielectric charging of the sample. A particular attention is paid to possible hysteresis phenomena that might arise due to the dielectric nature of the studied material. The modeling part of our work makes use of the well-known Dionne's model describing well the SEEY-curve parameters. ${ }^{20}$ It also considers the advancements in the TEEY modeling, made for bulk dielectrics in relation with the dielectric charging effect. ${ }^{21-26}$ Thus, after confirming the role of the dielectric layer thickness in the TEEY curve of thin $\mathrm{SiO}_{2}$ 
layers, a numerical model is developed to better understand the atypical shape of the TEEY curve of thin dielectric layers. The model accounts for the impact of Radiation Induced Conductivity (RIC), as well as for the contribution of the space-charge electric field induced by irradiation of the dielectric layer, and therefore for the Field Dependent Conductivity (FDC), to the TEEY curve in the studied energy range.

\section{EXPERIMENTAL PART}

\section{A. Sample elaboration}

The samples under study were thin silica $\left(\mathrm{SiO}_{2}\right)$ layers, $100 \mathrm{~nm}$-thick, thermally grown on Si-substrates. Intrinsic or heavily doped (p-type, Boron, resistivity $\rho_{S i}=0.002 \Omega \mathrm{cm}$ ) Silicon wafers, purchased from Sil'tronix, were used as substrates. Before being oxidized the Sisubstrates were chemically cleaned using Piranha solution (mixture of hydrogen peroxide $\left(\mathrm{H}_{2} \mathrm{O}_{2}\right)$ and sulfuric acid $\left.\left(\mathrm{H}_{2} \mathrm{SO}_{4}\right)\right)$. Then the ultrathin native silica layer covering the $\mathrm{Si}$ substrates, and known for its very poor dielectric properties, was removed by chemical etching in hydrofluoric acid (HF). Each step in the cleaning procedure was followed by rinsing in deionized water. Immediately after cleaning the Si-wafers were conditioned for the thermal $\mathrm{SiO}_{2}$ growth. The latter was performed at $1100^{\circ} \mathrm{C}$ under slightly oxidizing controlled atmosphere using a $\mathrm{N}_{2}-\mathrm{O}_{2}$ gas mixture containing $1.0 \%$ of $\mathrm{O}_{2}$. The $\mathrm{SiO}_{2}$ layers with targeted thicknesses were then obtained after HF-chemical etching of the obtained thermal $\mathrm{SiO}_{2}$. After etching the samples were rinsed in deionized water until attaining zero surface conductivity on the $\mathrm{SiO}_{2}$ thin layers.

\section{B. Structural and surface characterization of the $\mathrm{SiO}_{2}$ layers}

Structural characterization of the $\mathrm{SiO}_{2}$ layers was carried out by applying various diagnostic methods. The thicknesses of the $\mathrm{SiO}_{2}$ layers were determined by spectroscopic ellipsometry (SE) using a Sopra GES-5 ellipsometer in the $250-850 \mathrm{~nm}$ spectral range. The SE spectra were acquired with an incident angle of $75^{\circ}$, typical for Si-substrates. The recorded spectra were then modeled with Bruggeman's model ${ }^{27}$ to extract the $\mathrm{SiO}_{2}$ layer thicknesses. Optical data for the refractive index and the extinction coefficient, $\mathrm{n}$ and $\mathrm{k}$, provided by the SOPRA library, ${ }^{28}$ were used to perform the numerical analyses of the SE spectra.

Fourier Transform InfraRed (FTIR) spectroscopy has been performed for a structural analysis of the $\mathrm{SiO}_{2}$ layers before their thickness reduction. The used equipment was a Brucker Vertex 70 spectrometer. The spectra were recorded in a transmission mode. The latter was 
attainable due to the transparency to infrared light of the used intrinsic Si-substrates. The spectra were recorded under controlled atmosphere $\left(\mathrm{N}_{2}\right)$ in the energy range from 400 to $4000 \mathrm{~cm}^{-1}$ with a $2 \mathrm{~cm}^{-1}$ resolution. The transmittance spectra of the $\mathrm{SiO}_{2}$ layers were obtained after dividing the acquired spectra by the spectrum of a blank Si-substrate recorded under the same conditions and then converted to absorbance spectra for representation.

The TEEY is a phenomenon that is mainly affected by the first few or tens of nanometers beneath the surface. ${ }^{24}$ The contamination layer that usually builds up on the surface after air exposure has therefore a non-negligible impact on the TEEY. ${ }^{29}$ To limit contamination, the time between $\mathrm{SiO}_{2}$-sample preparation and TEEY measurements has been reduced to a minimum. The sample surface state was monitored by X-ray photoelectron spectrometry (XPS) to account for contamination. XPS measurements probe the first few nanometers of the sample, and thus provide information on the surface composition and contamination. The XPS electron source was mounted on the electron emission facility. The latter is equipped with an Omicron Nanotechnology EA125 hemispheric analyzer operating between $1 \mathrm{eV}$ and $2 \mathrm{keV}$, associated with an X-ray source. Once the samples are being installed in the vacuum chamber, both the XPS and the TEEY measurements were performed with no additional manipulation.

\section{Total Electron Emission Yield (TEEY) measurements}

\section{Experimental arrangement and methodology}

The TEEY curves were studied in the low energy range, using an electron gun Kimball ELG-2 operated in the energy range $1 \mathrm{eV}-2 \mathrm{keV}$ and provided the possibility of continuous or pulsed beam generation of the primary electrons (Fig. 2a). The measurements were performed under ultra-high vacuum $\left(5 \times 10^{-7} \mathrm{~Pa}\right)$. More details on the TEEY measurement facility can be found elsewhere. ${ }^{29,30}$

The applied for TEEY measurement methodology is based on simple considerations (Fig. 2). According to the current conservation law, the incident electron current $I_{0}$, the emitted current $I_{E}$ and the current $I_{S}$ that flows through the sample to the ground obey:

$$
I_{0}=I_{E}+I_{S} .
$$

For a given energy of the primary electrons, the TEEY measurements are performed in two steps: In a first step the incident electron current $I_{0}$ is determined (noted in blue on Fig. 2a). The sample holder is polarized at $+27 \mathrm{~V}$ which leads to collection of the low energy emitted electrons back to the dielectric layer surface due to the surface potential barrier. The measured current in this situation $I_{S}$ is very close to and is considered as $I_{0}{ }^{31}$ The emitted electron current 
$I_{E}$ is determined (in red on Fig. 2a) in a second step. During this step the sample holder is polarized at $-9 \mathrm{~V}$, impeding recollection of the emitted electrons. Knowing $I_{E}$ and $I_{0}$, the TEEY is deduced using the following expression:

$$
T E E Y=\frac{I_{E}}{I_{0}}=\frac{I_{0}-I_{S}}{I_{0}} .
$$

The TEEY curves are thus obtained over the whole energy range $0-2000 \mathrm{eV}$ and for different incident angles of the primary electron beam (Fig. 2b). Normal incidence is adopted as $0^{\circ}$ angle. The other reported incidence angles are measured with respect to the normal incidence.

(a)

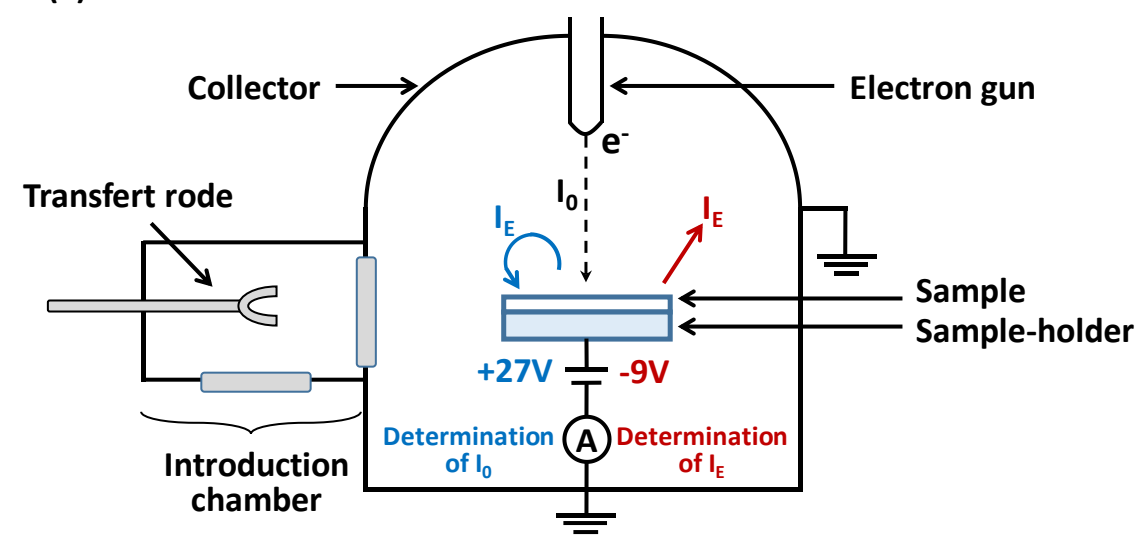

(b)

$$
\begin{gathered}
\text { Incident } \\
\text { angle }
\end{gathered}
$$

normal

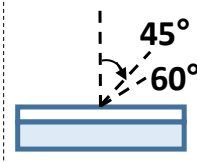

FIG. 2. (a) Schematic representation of the facility for TEEY and XPS measurements, (b) sketch of the incident angles of the electron gun used in the study; the reported angles are with respect to the normal incidence.

\section{Particular considerations when measuring the TEEY from dielectric materials}

Generally, the dielectric materials can locally trap electrical charges. Under irradiation, the primary electrons received by the dielectric, as well as the generated secondary electrons can be trapped after thermalization. Holes can get trapped following electron-hole pairs generation. Those trapped electrical charges impact the motion of incoming primary and outgoing secondary electrons through electrostatic interactions. According to the conventional total yield approach, ${ }^{22}$ when a continuous electron beam impacts a dielectric, the measured TEEY increases at first and then decreases to converge toward 1; an equilibrium state being reached with increasing the energy of the primary electrons. As the dielectric gets charged, the measured yield is no more representative of that of the neutral dielectric material. Therefore, in order to limit the dielectric charging, the use of pulsed electron beam is mandatory when evaluating the TEEY over the whole energy range. Those pulses should be short enough such that the injected charges do not influence further measurements. ${ }^{24}$ With using too long electron 
pulses, a hysteresis charging effect can be observed for the measured TEEY from dielectric materials, ${ }^{30}$ leading to a lower TEEY when repeating the experiment. For the TEEY measurements to be representative of the natural (uncharged) dielectric material, it is therefore appropriate to use minimum quantities of the primary electrons (short pulse durations) but sufficient ones to provide a favorable signal-to-noise ratio. In a previous work, we have found that for thin $\mathrm{SiO}_{2}$ layers, the pulses of primary electrons should be between 10 and $100 \mu \mathrm{s}$ duration, sent as a single pulse or in bursts of 10 pulses according to the resulting signal-tonoise ratio. ${ }^{30}$

For similar reasons the energy step should be chosen wisely too. A small energy step results in more measurement points over the entire energy range. However, such consideration increases the overall deposited electrical charge and influences the obtained results by lowering the measured TEEY. Step of around $20 \mathrm{eV}$ is small enough to precisely visualize the global shape of the TEEY curve while the dielectric charging is reduced to minimum. A smaller step, like $10 \mathrm{eV}$, provides more accurate information around the inflection points, but due to dielectric charging it lowers the nominal value of the measured TEEY and shifts its maximum toward lower energies.

\section{MODELING APPROACH}

To improve our understanding of the observed electron emission phenomena from thin dielectric layers, a 1D-model of TEEY has been developed. As mentioned earlier, our approach is based on the Dionne's model because of the reported good agreement between simulation and measurements of the SEEY from bulk $\mathrm{SiO}_{2}$ materials in the low energy range. ${ }^{20}$ Besides, the Dionne's model is related to physical considerations of the basic properties of the materials. It demonstrates that the first crossover energy $\mathrm{E}_{\mathrm{C} 1}$ can be very dependent on the surface properties of the material and the maximum yield is entirely controlled by the bulk properties of the material, in particular its conductivity and work function (in the case of insulators and semiconductors the sum of band gap and electron affinity). However, a detailed description of the TEEY from dielectric materials requires consideration of additional effects like charge build-up and space-charge electric field generation. The existent models in the literature for dielectrics account to some extent for these particular effects ${ }^{21-26}$ but similarly to Dionne's model they concern mainly bulk dielectrics and fail to explain the experimentally observed atypical shape of the TEEY curve of thin dielectric layers. 


\section{A. Dionne's model providing the typical shape of Secondary Electron Emission Yield} curve

According to the Dionne's model ${ }^{20}$ the SEEY can be expressed by the product of three distinct contributions:

$$
\mathrm{SEEY}=G . T \cdot B
$$

where the $G$-term represents the generation of secondary electrons, the $T$-term describes the transport of secondary electrons from their generation point to the surface of the material and the $B$-term provides the probability of secondary electrons to escape the material. Consideration of these three contributions suffices to represent the physical situation of electron emission from metals. Schematically they are represented in Fig. 3a, including the part accounting for backscattered electrons.

(a)

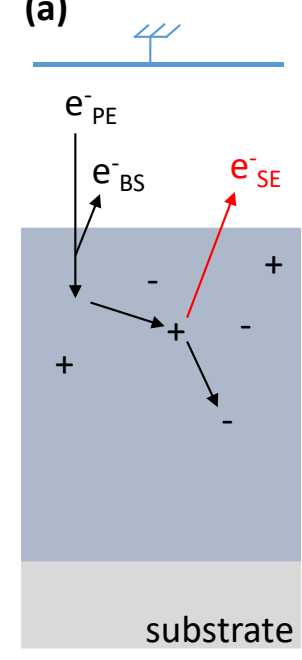

(b)

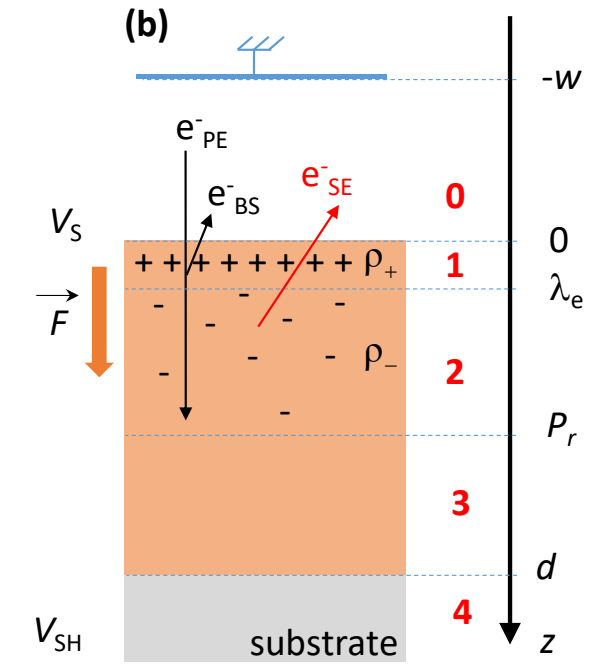

(c)

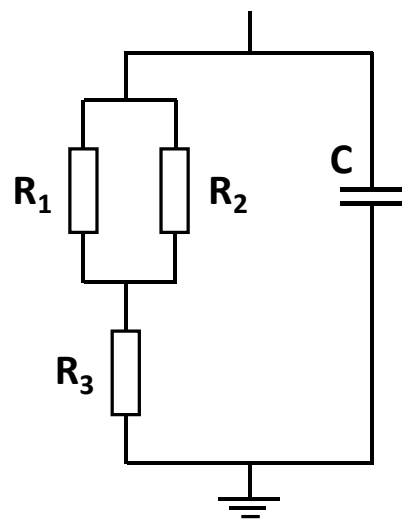

FIG. 3. Schematic representation of the charge distribution during electron irradiation: (a) Dionne's model and (b) proposed in this work Dionne's model adapted to thin dielectric layers and (c) equivalent circuit of the processes of electron irradiation in a thin $\mathrm{SiO}_{2}$ layer.

The generation of secondary electrons ( $G$-term) depends on the absorption of the energy of primary electrons, accounting also for their maximum penetration depth $P_{r}(E)$ :

$$
G=\frac{1}{\xi}\left(A_{a} b \lambda_{\mathrm{e}}\right)^{\frac{1}{b}}\left(\frac{P_{r}}{\lambda_{\mathrm{e}}}\right)^{\frac{1}{b}-1},
$$

where $\xi$ is the ionization energy that can be approximated by the work function if the material is a metal and by the $\xi=\chi+E_{g}$, if it is a semiconductor or a dielectric, with $\chi$ being the electron affinity and $\mathrm{E}_{\mathrm{g}}$ the gap energy of the dielectric material. $A_{\mathrm{a}}$ is the energy absorption coefficient of primary electrons, related to the material density. ${ }^{32}$ The energy of primary electrons is 
absorbed in the material according to a power law with $b$ being its exponent $(b>1)$, set to 1.35 by experiments. ${ }^{20}$ The quantity $\lambda_{\mathrm{e}}$ represents the mean escape depth of secondary electrons, which is actually considered equal to the mean free path of electrons in the material in the Dionne's model.

The maximum penetration depth of primary electrons depends on their energy $P_{r}(E)$ through the energy-range relation: ${ }^{33}$

$$
P_{r[\mathrm{~nm}]}(E)=\frac{A}{\rho_{m\left[\mathrm{~g} / \mathrm{cm}^{3}\right]}} E_{[\mathrm{keV}]}^{b},
$$

where $\rho_{m}\left[\mathrm{~g} / \mathrm{cm}^{3}\right]$ is the mass density of the dielectric, $A$ is a constant, with the appropriate unit, characteristic of the material and related to the stopping power for the $\mathrm{SiO}_{2}{ }^{33}$ The stopping power is larger for heavy materials, like $\mathrm{Au}, \mathrm{Pt}, \mathrm{Pb}$, etc., and much smaller for light materials, as for the studied here $\mathrm{SiO}_{2}$. The constant $A$ is calculated here for $\mathrm{SiO}_{2}(A=98)$. The calculation is based on Bethe's equation ${ }^{34}$ and considering relativistic, and Nguyen-Truong ${ }^{35}$ corrections. Bethe's corrected equation provides the stopping power of a material while taking into account the energy of primary electrons, the mean excitation energy and the atomic number of the material. The power factor $b$ is considered constant, equal to 1.35 , in the entire energy range. ${ }^{36,37}$

The transport of secondary electrons ( $T$-term) is expressed by the probability of a secondary electron, generated between the maximum penetration depth of primary electrons $P_{r}(E)$ and the material surface, to reach the surface:

$$
T=1-e^{-\frac{P_{r}}{\lambda_{\mathrm{e}}}}
$$

The escape of secondary electrons, i.e., their possibility to overcome the energy barrier at the material surface $(B$-term) is expressed by:

$$
B=\left(1-\sqrt{\frac{\chi}{\overline{\mathrm{E}_{\mathrm{SE}}}}}\right) .
$$

The escape probability is related to the to the fraction of transmitted secondary electrons via their incident energy $\overline{\mathrm{E}_{\mathrm{SE}}}$. The remaining fraction of secondary electrons is considered reflected..$^{38}$ Finally, the SEEY is expressed by:

$$
\mathrm{SEEY}=G \cdot T \cdot B=\left[\frac{1}{\xi}\left(A_{a} b \lambda_{\mathrm{e}}\right)^{\frac{1}{b}}\left(\frac{P_{r}}{\lambda_{\mathrm{e}}}\right)^{\frac{1}{b}-1}\right]\left[1-e^{-\frac{P_{r}}{\lambda_{\mathrm{e}}}}\right]\left[1-\sqrt{\frac{\chi}{\overline{\mathrm{E}_{\mathrm{SE}}}}}\right] .
$$

It gives the relation between the parameters of the SEEY curve and the variables which describe some of the electronic and chemical properties of the material. 


\section{B. This work proposal: Dionne's model adapted to thin dielectric layers}

In an attempt to gain further insight in the TEEY curve of thin dielectric layers, it is appropriate to examine more carefully some of the quantities of Eq. (8). To that end we have adapted the Dionne's model to thin dielectric layers. In the proposed here model the studied sample is represented by a stratified structure (Fig. 3b), following the main lines in some of the earlier works on TEEY from dielectrics reported in the literature. ${ }^{21-24,26}$ As demonstrated in those works, a stratified structure of the model leads to a straightforward expression of the surface potential $V_{\mathrm{S}}$ which can be experimentally measured. ${ }^{22}$ The stratified model, alongside with the electrostatic equations, successfully explains the sign of the surface potential for the first moment of irradiation, as well as in steady state. It also leads to a better explanation of the nominal value of TEEY and of the position in energy for the second crossover $\left(\mathrm{E}_{\mathrm{C} 2}\right)$, including its disappearance for some materials. Moreover, it emphasizes the necessity to consider the interaction of incoming (primary) electrons with previously trapped charges. Such consideration is made in this work to express the space-charge electric field generated from the positive layer near the surface and the negative layer beneath it, and to evaluate its impact on the TEEY.

The proposed here model is $1 \mathrm{D}$, because the $\mathrm{SiO}_{2}$-layers under study are very thin (less than $100 \mathrm{~nm}$ ), i.e., their thicknesses are much smaller than their lateral dimensions and the irradiated area (diameter of the spot $4 \pm 1 \mathrm{~mm}$ ). One can consider in this case that no lateral effects significantly impact the electron emission. Such assumption keeps the model simple and the computational time very short, however providing the essential information on TEEY. Limiting the system describing the irradiated sample to one dimension is an approximation that already has been approved by other authors. ${ }^{23,26}$

In order to compare with experimentally obtained TEEY curves, the proposed here model includes the backscattered electron emission yield (BEEY), which is considered constant in the studied energy range, with a fraction of $20 \%(\eta=0.2) .{ }^{39}$ Finally, the TEEY is obtained from:

$$
\mathrm{TEEY}=\mathrm{BEEY}+\mathrm{SEEY}
$$

The working hypothesis of the model is that the secondary electrons are originated from approximatively the first $10 \mathrm{~nm}$ beneath the surface. Consequently, this region can be considered as populated in majority by positive charges. Below this layer, the primary electrons are trapped, and the secondary electrons are too far from the surface to be emitted, so the 
corresponding layer is populated in majority by negative charges. Thus, the physical situation that we wish to describe, including the studied dielectric layer of thickness $d$, can be represented by 5 media (numbered from 0 to 4, in Fig. 3b). The vertical axis in Fig. 3b indicates the depth within the dielectric layer, with $z=0$ corresponding to the surface of the $\mathrm{SiO}_{2}$ layer:

- Medium 0 is the vacuum separating the primary electron source (electron gun) from the $\mathrm{SiO}_{2}$ surface.

- Medium 1 is delimited by the surface of the sample and the mean escape depth of secondary electrons $\lambda_{\mathrm{e}}$. This is the region where the charge density is positive (noted $\left.\rho_{+}\right)$. Accumulation of electrical charges leads to the build-up of an electric field inside the dielectric material and at its surface. This space-charge field drives the charges in the dielectric, and may notably impact the transport of secondary electrons toward the surface as it appears accelerating for them. Field dependent effects should therefore be included in the model. Previous works addressed different aspects of the contribution of an electric field to the SEEY. ${ }^{40,41}$ Through Monte-Carlo calculations based on the Fröhlich theory, Fitting and Boyde ${ }^{40}$ have studied the modification of secondary electrons emission when $\mathrm{SiO}_{2}$ material is subjected to an external electric field $F$ (positive or negative). It has been established that the mean escape depth of secondary electrons increases exponentially in field direction $(F>0)$ and diminishes in opposite direction $(F<0)$. In the electric field range $-2 \times 10^{8}-+2 \times 10^{8} \mathrm{~V} / \mathrm{m}$ the field dependence of $\lambda_{\mathrm{e}}$ follows the general rule:

$\lambda_{\mathrm{e}}(F)=\lambda_{\mathrm{e} 0} e^{-\beta F}$,

where $\lambda_{\mathrm{e} 0}[\mathrm{~nm}]$ is the mean free path of electrons inside $\mathrm{SiO}_{2}$ with no electric field and $\beta$ is an experimentally determined constant, taking value of $\beta=3.6 \times 10^{-9}[\mathrm{~m} / \mathrm{V}]$ for $\mathrm{SiO}_{2} \cdot{ }^{40}$ Such electric field dependence is implemented in our model to account for the variation of the escape depth of secondary electrons $\lambda_{\mathrm{e}}$ as function of the space-charge generated electric field. As previously suggested, ${ }^{42}$ additional contributions like lowering of the Schottky barrier at the interface material/vacuum and the electrons/holes recombination ${ }^{43}$ constitute second order effects and may be neglected in a first approximation for the low energy range of primary electrons when the surface of the studied samples is flat. The energy range of primary electrons applied here $(E<2 \mathrm{keV})$ and the good flatness of the studied $\mathrm{SiO}_{2}$ samples (roughness $R_{a}<1 \mathrm{~nm}^{44,45}$ ) perfectly meet these requirements. 
- Medium 2 extends from the mean escape depth of secondary electrons $\lambda_{\mathrm{e}}(F)$ to the maximum depth reached by the primary electrons $P_{r}(E)$. In this zone, the negative charges form the majority (the charge density being noted $\rho_{\text {-). }}$. The cumulated charged part of the dielectric layer thus increases with the penetration depth of the primary electrons $P_{r}(E)$ and is directly related to the energy of incident electrons via the energyrange relation Eq. (5).

- Medium 3 expands beyond the maximum penetration depth of primary electrons $P_{r}(E)$ down to the substrate, i.e., it involves the non-irradiated part of $\mathrm{SiO}_{2}$. This part might entirely disappear for very thin samples, for which the maximum penetration depth of primary electrons is comparable to their thickness.

- Medium 4 is the substrate (highly doped silicon wafer in this work).

The electrostatic equations in each medium are derived from Poisson's equation:

$$
\operatorname{div}(F)=\frac{\rho}{\varepsilon_{0} \varepsilon_{r}}=-\nabla^{2} V,
$$

applying the boundary conditions at the interfaces between the different media. Thus, we obtain expressions for the potential and electric field at each position $z$ in depth of the $\mathrm{SiO}_{2}$ layer. In Eq. (11) $\rho$ is the total charge density, $\varepsilon_{0}$ is the vacuum permittivity and $\varepsilon_{r}$ is the relative permittivity of the dielectric. The collector, located at position $z=-w$, is connected to the ground defining $V_{\text {coll }}(-w)=0 \mathrm{~V}$, while the sample holder is polarized with potential $V_{\mathrm{SH}}(d)=-9 \mathrm{~V}$. The electric field (indexed to the corresponding medium) as function of the position $z$ is written:

$$
\begin{array}{ll}
F_{0}(z)=F_{c}, & \text { for } \quad-w \leq z \leq 0 \\
F_{1-3}(z)=\frac{1}{\varepsilon_{0} \varepsilon_{r}} \int_{0}^{z}\left(\rho_{+}\left(z^{\prime}\right)+\rho_{-}\left(z^{\prime}\right)\right) d z^{\prime}+\frac{F_{c}}{\varepsilon_{r}}, & \text { for } \quad 0 \leq z \leq d \\
F_{4}(z)=0 &
\end{array}
$$

$F_{c}$ is determined based on the condition for the potential set to the holder:

$$
-V_{S H}=\int_{0}^{d} F\left(z^{\prime}\right) d z^{\prime}+F_{c}\left(w+\frac{d}{\varepsilon_{r}}\right) \quad \text { for } \quad z>d
$$

In addition to the above described phenomena of electron irradiation of dielectrics, we take into account in the model the phenomenon of Radiative Induced Conductivity (RIC). Our reasoning to consider RIC in the model relies on balance considerations: the model shall take into account competition between charging and discharging processes. The RIC, occurring in Media 1 and 2 (Fig. 3b), favors a discharge of the irradiated sample. In general, RIC depends on the interaction between an ionizing radiations and a dielectric material, as well as on the 
intrinsic electrical properties of the dielectric. The increase in conductivity of a dielectric material under irradiation is given by: ${ }^{46,47}$

$$
\sigma_{\mathrm{RIC}}=\sigma_{\mathrm{SiO}_{2}}\left(\frac{d D}{d t}\right)^{\Delta_{\mathrm{p}}}
$$

where $\sigma_{\mathrm{SiO} 2}$ is the conductivity of the material without irradiation, $D$ is the radiation dose (calculated from the averaged deposited energy over the maximum penetration depth of the electrons) and $d D / d t$ is the radiation dose rate. The power factor $\Delta_{\mathrm{p}}$ in Eq. (16) is an unitless parameter. It is related to the shape of the charge traps distribution in dielectric materials. It depends on the nature of the dielectric material and varies in the range $0.5-1 .{ }^{46}$ For the $\mathrm{SiO}_{2}$ layers, studied in this work, it is estimated to $\Delta_{\mathrm{p}}=0.7$, based on the methodology developed for charge properties of dielectric materials applied for space devices. ${ }^{47}$

Because of the space-charge effect, the generated electric field inside the dielectric material can be very high. In order to account for the evolution of the conductivity of the studied material as a function of the space-charge field, i.e., the field dependent conductivity (FDC), the applied expression considers non-linear charge transport mechanisms, among others the Poole-Frenkel formalism. ${ }^{48}$ Beyond a threshold electric field, $F_{t h}$, The FDC reads:

$$
\begin{array}{ll}
\sigma_{F D C}=\sigma_{S i O_{2}}=1 \times 10^{-16} \mathrm{Sm}^{-1} & \text { for } F<F_{t h} \\
\sigma_{F D C}=\sigma_{S i O_{2}} \exp \left(\frac{\beta_{F D C} \sqrt{F-F_{t h}}}{k T}\right) & \text { for } F>F_{t h}
\end{array}
$$

with $\beta_{F D C}=\sqrt{\frac{q^{3}}{\pi \varepsilon_{0} \varepsilon_{r}}}$ and $F_{t h}=2 \times 10^{8} \mathrm{~V} / \mathrm{m}$, representing the threshold of non-linear electric field behaviour, as measured for $\mathrm{SiO}_{2}$-thin layers. ${ }^{49}$ The other quantities in relation (18) are as follows: $q$ is the elementary charge, $k$ is the Boltzmann constant and $T$ is the temperature, considered here $T=300 \mathrm{~K}$. Without loss of generality, the constant $\beta_{F D C}$ is defined here as intermediate between the constant for the Schottky limit and the Poole-Frenkel constant accounting for the electronic part of the relative permittivity of the dielectric material, moreover that the difference between the electronic part of the relative permittivity of $\mathrm{SiO}_{2}, \varepsilon_{\infty}=2.24,{ }^{50,51}$ as extracted from SE measurements and the low-frequency part of it, $\varepsilon_{r}=3.9$, as defined at $1 \mathrm{kHz},{ }^{51,52}$ is small. Such consideration implies multiple-type field dependent conductivity in the dielectric material. ${ }^{48}$

To represent the above described processes an equivalent circuit accounting for the electron irradiation of a thin $\mathrm{SiO}_{2}$ layer is given in Fig. 3c. The region where RIC and FDC are 
occurring extends until the maximum penetration depth of primary electrons, $P_{r}(\mathrm{E})$. This region is characterized by a resistance $R_{1,2}$, while the resistance of the remaining, non-irradiated part of the dielectric layer is given by $R_{3}$, with the sum of both defining the equivalent $R_{e q}$ of the circuit:

$$
R_{1,2}=\frac{1}{\sigma_{R I C}+\sigma_{F D C}} \frac{P_{r}}{S}, R_{3}=\frac{1}{\sigma_{F D C}} \frac{\left(d-P_{r}\right)}{S}, \quad R_{e q}=R_{1,2}+R_{3},
$$

where $S$ represents the irradiated by electrons area. The capacitance in the circuit is calculated according:

$$
C=\varepsilon_{0} \varepsilon_{r} \frac{S}{d} .
$$

The irradiated area considered here is of $S=12.6 \mathrm{~mm}^{2}$ which gives a value of the capacitance in the order of $\mathrm{nF}$ depending on the $\mathrm{SiO}_{2}$ layer thickness $(C=4.82 \mathrm{nF}$ for $d=90 \mathrm{~nm})$.

Flow-chart of the proposed Dionne's model adapted to thin dielectric layers is shown in Fig. 4 and all required parameters are summarized in the Table below. Input parameters of the model are the electron beam current density, the pulse duration and the initial energy of the incident electrons $E_{0}$. As shown in the flowchart the model starts with initialization of the variables; $i$ is an integer, accounting for the step increase in the energy of primary electrons to obtain the TEEY curve in the entire energy range.

The numerical procedure involves the following steps:

(1) Determination of the charge source

For a given energy of the primary electrons, from the electron gun parameters, i.e., beam current and pulse duration, the quantity of primary electrons $\left(\mathrm{Q}_{0}\right)$ is determined. Based on their energy, the maximum penetration depth of the primary electrons $\left(P_{r}(E)\right)$ is found after Eq. (5).

(2) Estimation of the charge density and the space-charge electric field

Once a pulse of electrons reaches the dielectric layer, a fraction of them is considered backscattered, the remaining part penetrates the layer. The secondary electron emission associated with the electron pulse produces a variation of the trapped positive charge, calculated using the TEEY obtained for the current energy value and existing charge distribution and escape depth. For the energy $E_{0}$ the initial TEEY 0 corresponds to the one extracted from the Dionne's model with no electric field (Eq. (8)). The positive charge density is considered constant in the region $\left[0-\lambda_{\mathrm{e}}\right]$. Part of the trapped positive and negative charges is evacuated to the substrate by conduction, involving notably RIC and FDC nonlinear mechanisms (Eqs. (16) - (18)). New charge densities $\rho_{+}^{\prime}$ and $\rho^{\prime}$ - are produced, considering: 


$$
\rho_{ \pm}^{\prime}=\rho_{ \pm} \exp \left(-\frac{\Delta t}{\tau}\right),
$$

where $\tau$ is the time constant of the RC-circuit (Fig. 3c), defined by Eqs. (19) - (20). The electric field distribution is calculated then from $\rho^{\prime}+$ and $\rho^{\prime}$ - using Eqs. (12) - (15).

(3) Mean escape depth and TEEY

Then, the mean escape depth of secondary electrons, function of the space-charge electric field, is calculated from Eq. (10), using the maximum of the space-charge electric field near the dielectric surface. Finally, the TEEY function of energy is computed via eq. (9). The energy is increased by an amount $\Delta \mathrm{E}$ and the loop is repeated. At each step, the energy of the primary electrons is increased and another pulse of electrons is sent.

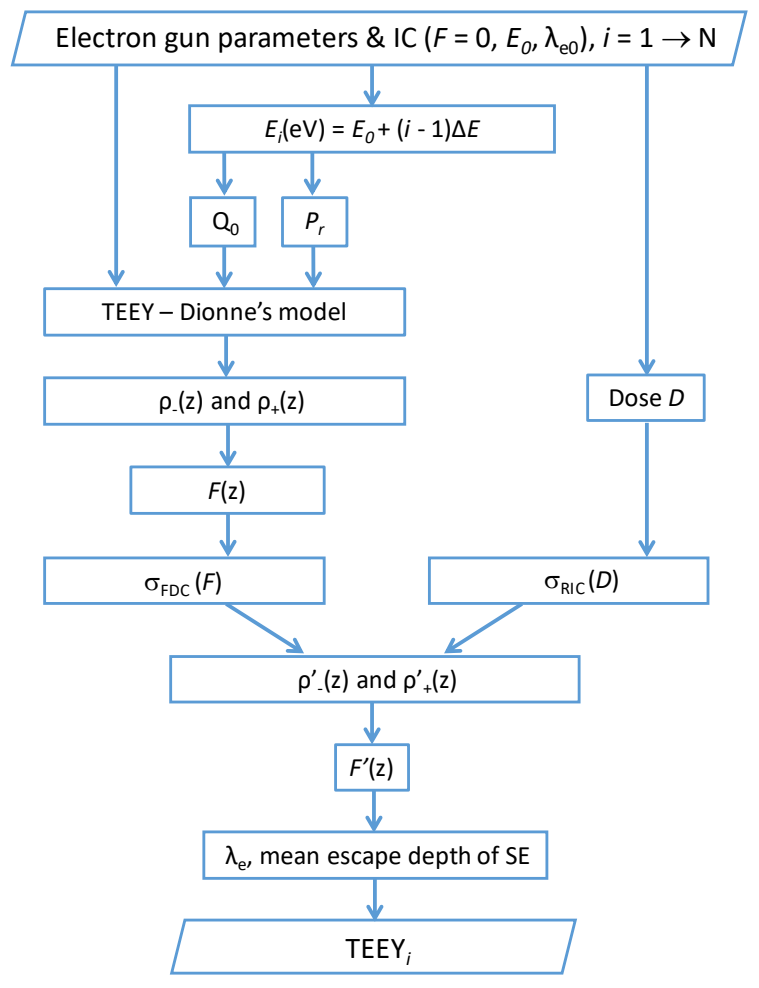

FIG. 4. Flow-chart for the Dionne's model adapted for thin dielectric layers.

The output quantities from the here presented Dionne's model adapted for thin dielectric layers are the TEEY curve in the entire energy range of primary electrons, the distribution of the electric field induced by space-charge for a given energy of the primary electrons and the averaged escape depth of secondary electrons. Other quantities can be extracted at intermediate steps. This simple simulation is performed on Matlab ${ }^{\circledR}{ }^{53}$ in few seconds and does not require high computational resources. 
Table. List of quantities and parameters used in the Dionne's model adapted for thin dielectric layers

\begin{tabular}{|c|c|c|c|}
\hline Quantity/Parameter & Value & Nature & Reference \\
\hline$d$-sample thickness & $10-100 \mathrm{~nm}$ & Experimental & This work \\
\hline$\varepsilon_{r}$ - relative permittivity of $\mathrm{SiO}_{2}$ & 3.9 & Experimental & 51 \\
\hline $\mathrm{E}_{\mathrm{g}}$ - energy of optic gap of $\mathrm{SiO}_{2}$ & $9.0 \mathrm{eV}$ & Experimental & 51 \\
\hline$\chi$ - electron affinity of $\mathrm{SiO}_{2}$ & $0.9 \mathrm{eV}$ & Experimental & 54 \\
\hline$\sigma_{\mathrm{SiO} 2}$ - low field conductivity of $\mathrm{SiO}_{2}$ & $10^{-16} \mathrm{Sm}^{-1}$ & Experimental & 54 \\
\hline$\rho_{\mathrm{m}}-$ mass density of $\mathrm{SiO}_{2}$ & $2.33 \mathrm{~g} / \mathrm{cm}^{3}$ & Experimental & 54 \\
\hline$A_{a}-$ absorption coefficient of primary electrons & $40.8 \mathrm{eV}^{\mathrm{b}} / \mathrm{nm}$ & Estimation & This work \\
\hline$b$ - power factor in Eqs. (4) and (5) and (8) & 1.35 & Experimental & 20 \\
\hline$\overline{\mathrm{E}_{\mathrm{SE}}}$ - mean energy of secondary electrons & $7.0 \mathrm{eV}$ & Theoretical & 23 \\
\hline$\eta$ - fraction of backscattered electrons - BEEY & 0.2 & Estimation & 39 \\
\hline $\begin{array}{l}\lambda_{\mathrm{e} 0} \text { - mean free path of electrons inside } \mathrm{SiO}_{2} \text { with no } \\
\text { electric field, Eq. (10) }\end{array}$ & $7.0 \mathrm{~nm}$ & Theoretical & 55 \\
\hline$\beta$ - coefficient of attenuation in Eq. (10) & $3.6 \times 10^{-9} \mathrm{~m} / \mathrm{V}$ & Theoretical & 40 \\
\hline$\Delta_{\mathrm{p}}-\mathrm{RIC}$ power factor in Eq. (16) & 0.7 & Estimation & This work \\
\hline$F_{t h}-$ Field threshold for non-linear conductivity & $2 \times 10^{8} \mathrm{~V} / \mathrm{m}$ & Experimental & 49 \\
\hline $\mathrm{I}_{0}$ - incident current & $2.0 \mu \mathrm{A}$ & Experimental & This work \\
\hline $\mathrm{S}$ - irradiated surface & $12.6 \mathrm{~mm}^{2}$ & Experimental & This work \\
\hline$\Delta \mathrm{t}-$ pulse duration & $100 \mu \mathrm{s}$ & Experimental & This work \\
\hline$h$ - distance e-gun to sample & $5 \mathrm{~cm}$ & Experimental & This work \\
\hline$w$ - distance collector-sample & $10 \mathrm{~cm}$ & Experimental & This work \\
\hline$V_{S H}$ - potential of the sample holder & $-9,-18$ or $-27 \mathrm{~V}$ & Experimental & This work \\
\hline$V_{\text {coll }}$ - potential of the collector & $0-800 \mathrm{~V}$ & Experimental & This work \\
\hline
\end{tabular}

\section{RESULTS AND DISCUSSION}

\section{A. Thickness, structural characterization and surface state of the thermal $\mathrm{SiO}_{2}$ layers}

As the current study is on the TEEY from very thin dielectric layers, $\mathrm{SiO}_{2}$-samples with thicknesses in the range $10-100 \mathrm{~nm}$ were prepared and used. Tight control on the thickness and structural properties of the $\mathrm{SiO}_{2}$ layers is essential to evaluate the dependence of TEEY on the dielectric layer thickness, especially for very thin layers, like the ones studied here. It strengthens the analysis on different mechanisms and their contributions to the measured TEEY. Moreover, it is important to control the layer thickness over a surface larger than the irradiated area defined by the spot of the primary electron beam to verify the assumption made for the 1D-model developed here. To that end, the $\mathrm{SiO}_{2}$-layer thickness was measured, for each sample, using spectroscopic ellipsometry on the entire surface, using mapping. 
Figure 5 shows the layer thickness obtained by spectroscopic ellipsometry (SE) for one of the as-grown $\mathrm{SiO}_{2}$ layers. Map of the point distribution, used to scan the layer, is given on Fig. 5a. There are 37 points arranged in polar coordinates with a step of $\Delta r=7 \mathrm{~mm}$ in radius and an angle $\Theta=30^{\circ}$. The solid black line shows the sample limits. Such organization of the scanning point provides less points in the sample periphery but more points in the central part where characterization of the TEEY curve is performed. The thickness map reported in Fig. $5 b$ applies only for the scanned region. The reported value of the thickness is averaged over the measured surface. The obtained averaged thickness of this $\mathrm{SiO}_{2}$ layer is $d=\overline{120.6} \pm 1.5 \mathrm{~nm}$, revealing a low thickness dispersion of the $\mathrm{SiO}_{2}$ layers with only $3 \mathrm{~nm}$ difference between the lowest and the highest values over a surface of $13.85 \mathrm{~cm}^{2}$. Given the very small area of the electron beam spot $\left(S=0.126 \mathrm{~cm}^{2}\right)$ one can consider that the thickness of $\mathrm{SiO}_{2}$ layers under the irradiated area is identical even for the very thin layers.
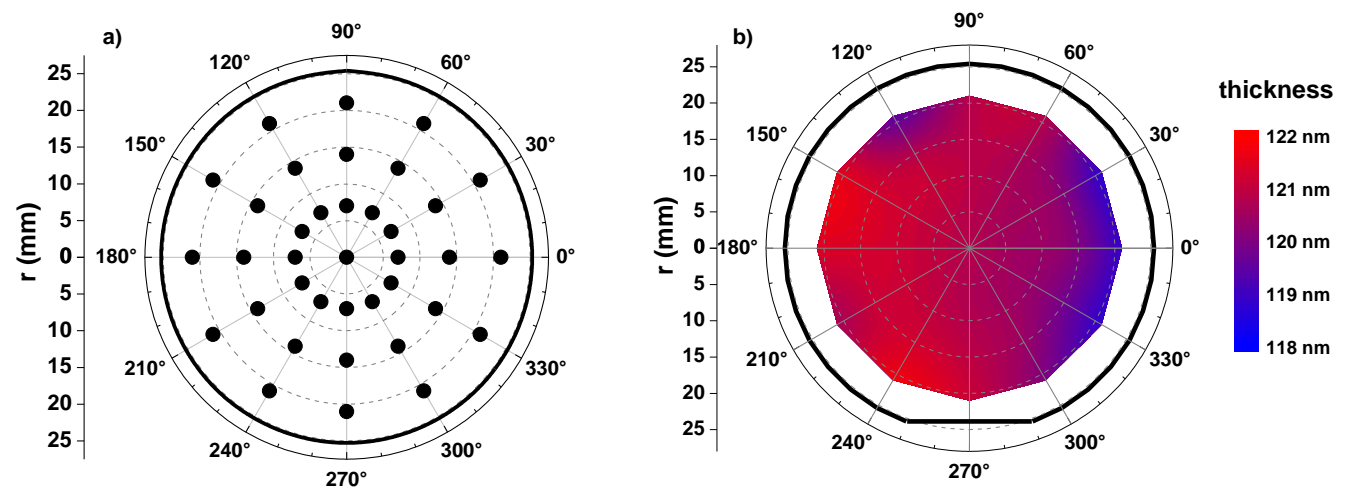

FIG. 5. (a) Measurement point distribution in polar coordinates; the solid black line shows the Si-wafer limits and (b) thickness map of an as-grown $\mathrm{SiO}_{2}$ layer with averaged, over the sample surface, thickness of $d=\overline{120.6} \pm 1.5 \mathrm{~nm}$; to easily locate the zones of further measurements for all measured $\mathrm{SiO}_{2}$ samples the Sisubstrate was positioned with the primary flat at $270^{\circ}$.

Ellipsometric angles $(\tan (\Psi)$ and $\cos (\Delta))$ of the recorded spectra (dots) for four different $\mathrm{SiO}_{2}$ layers, after reduction of their thicknesses to the targeted ones, are shown in Fig. 6 where the SE measurements apply for the central point of each sample. The proceeded spectra (lines) are presented on the same figure as well. The deduced sample thicknesses are reported on the figure. As it can be noticed, the goodness of the fit is very high (confidence level $R^{2}=0.999$ ) for all measurements, with a standard deviation of the $\mathrm{SiO}_{2}$-layer thickness of only $\pm 0.1 \mathrm{~nm}$. 


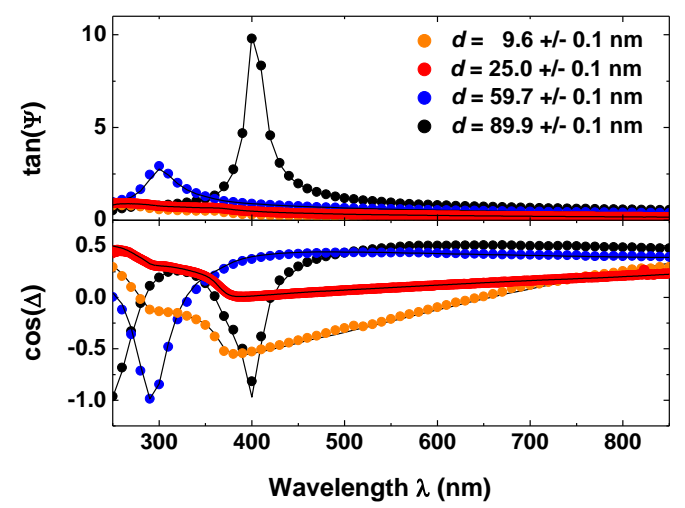

FIG. 6. Recorded ellipsometric spectra (dots) and Bruggeman's simulated spectra (lines) for the studied $\mathrm{SiO}_{2}$ layers.

Structural characterization of the as-grown thermal $\mathrm{SiO}_{2}$ layers was performed by FTIR spectroscopy in transmission mode and one of the recorded spectra is shown in Fig. 7. We recall here that the FTIR measurements were performed on the $\mathrm{SiO}_{2}$-layers grown on intrinsic Sisubstrates, because of the transparency of the latter to infrared light. The obtained spectrum (Fig. 7) is typical for $\mathrm{SiO}_{2}$ material. ${ }^{56,57}$ One can clearly see the three major transverse-optic (TO) vibrational modes which are characteristic of $\mathrm{SiO}_{2}$. Each of these three TO absorption bands can be described by a particular vibrational mode of the oxygen $(\mathrm{O})$ atoms with respect to the paired silicon $(\mathrm{Si})$ atoms, necessary to form the bridge $\mathrm{Si}-\mathrm{O}-\mathrm{Si}$. The lowest-frequency TO band centered at $457 \mathrm{~cm}^{-1}$ in the spectrum in Fig. 7 characterizes the rocking (R) vibrational behavior of the $\mathrm{O}$-atom above an axis passing through the two $\mathrm{Si}$ atoms. Symmetrical stretching (SS) of the O-atom along a line crossing the axis formed by the two Si atoms characterizes the vibrational mode of the middle TO band centered at $810 \mathrm{~cm}^{-1}$. The third TO band centered at higher frequency $1079 \mathrm{~cm}^{-1}$, belongs to an asymmetrical stretch (AS) motion in which the Oatom moves back and forward along a line parallel to the axis through the two Si atoms. The AS vibration actually gives rise to two modes: 1) an AS mode in which adjacent $\mathrm{O}$-atoms execute the AS motion in phase with each other, and 2) an AS mode in which adjacent O-atoms move at $180^{\circ}$ out of phase with each other. Deconvolution of this AS vibration (insert in Fig. 7) allows to distinguish the two components, the main peak at $1079 \mathrm{~cm}^{-1}$ and a shoulder positioned at $1200 \mathrm{~cm}^{-1}$. The narrow band peaking at $1079 \mathrm{~cm}^{-1}$ has a Full-Width Half-Maximum (FWHM) of only $76 \mathrm{~cm}^{-1}$ indicating that the as-grown thermal $\mathrm{SiO}_{2}$ layers are structurally well organized. The obtained in this study spectral positions of the three TO vibrational modes, as well as the value of FWHM of the AS band are in full agreement with values reports in the literature for thermally grown $\mathrm{SiO}_{2}$ layers. ${ }^{56,57}$ 


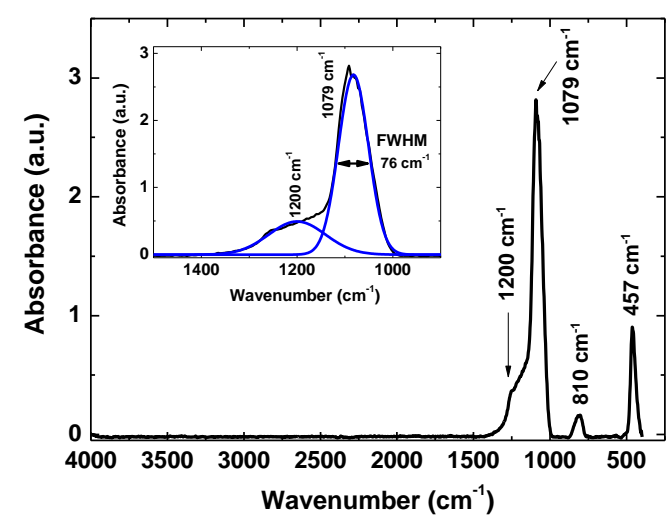

FIG. 7. FTIR spectrum of $\mathrm{SiO}_{2}$ layer (thickness of $91.5 \mathrm{~nm}$ ). Insert: deconvolution of the asymmetric stretching peak at $1079 \mathrm{~cm}^{-1}$ and the shoulder $1200 \mathrm{~cm}^{-1}$.

Evaluation of the surface state of the studied $\mathrm{SiO}_{2}$ layers is of utmost importance since the electron emission is considered to originate mainly from the first $10 \mathrm{~nm}$ beneath the layer surface. The performed XPS measurements, in order to check for potential contamination of the $\mathrm{SiO}_{2}$-layer surface, were realized shortly after reduction of the $\mathrm{SiO}_{2}$ layer thickness and just after positioning the sample in the facility to make the TEEY measurement. It is worth recalling here that the XPS system is an integral part of the facility for TEEY measurements and allows for correlation between of surface state analyses and TEEY measurements. The few minutes of exposure of the samples to ambient air, after their elaboration, actually do not cause contamination of the $\mathrm{SiO}_{2}$-layer surface that may deviate the TEEY. The carbon peaks in the obtained XPS survey spectrum (not shown) are indistinguishable from the background noise. Their intensity is less than $0.5 \%$ of the detection threshold of the XPS configuration.

\section{B. TEEY of thin $\mathrm{SiO}_{2}$ layers}

\section{Influence of $\mathrm{SiO}_{2}$ thickness on the TEEY}

To study the influence of the dielectric layer thickness on the TEEY for thin $\mathrm{SiO}_{2}$ layers, the four samples presented earlier were selected. The thinnest one is of only $9.6 \mathrm{~nm}$. Such thickness is very close to the mean free path of electrons in $\mathrm{SiO}_{2}, \lambda_{\mathrm{e} 0}=7.0 \mathrm{~nm}{ }^{55}$ The second layer is $25.0 \mathrm{~nm}$ thick. Thickness of the dielectric layers in the range $25-30 \mathrm{~nm}$ is usually associated with a transition in their structural and optical properties toward the characteristic ones of bulk materials. The two other $\mathrm{SiO}_{2}$-samples are with thicknesses of $59.7 \mathrm{~nm}$ and $89.9 \mathrm{~nm}$. These are thicknesses of dielectric layers typically used for insulation and cover layers in electronic devices, with the latter one presenting antireflective properties, in addition. ${ }^{44}$ 
Measured TEEY curves of these four $\mathrm{SiO}_{2}$-layers are presented in Fig. 8 for the energy range of primary electrons $0-2000 \mathrm{eV}$. All four curves reveal behaviour different from the typical shape of TEEY (Fig. 1). Instead of a quick increase of TEEY, followed by a maximum for energies above the first crossover energy $\mathrm{E}_{\mathrm{C} 1}$ and then a slow decrease for higher energies of the primary electrons, the TEEY curve presents an atypical shape with a dip (local minimum) after the first crossover energy $\mathrm{E}_{\mathrm{C} 1}$ and then an increase to attain a shape identical to the typical TEEY curve. It is worth noticing here that the atypical shape of TEEY has only been observed for thin dielectric layers (order of $100 \mathrm{~nm}$ and less). Moreover, the TEEY shape depends on the dielectric thickness; the atypical shape of the TEEY curve is less pronounced for the very thin $\mathrm{SiO}_{2}$ layers $\left(9.6 \mathrm{~nm}\right.$ and $25.0 \mathrm{~nm}$ ) compared to the thin $\mathrm{SiO}_{2}$ layers $(59.7 \mathrm{~nm}$ and $89.9 \mathrm{~nm})$. The interception value and the slope of TEEY at the first crossover energy $\mathrm{E}_{\mathrm{C} 1}$ are identical for all samples, revealing identical surface state (composition and contamination level) of the $\mathrm{SiO}_{2}$ layers. Except for the $25.0 \mathrm{~nm}$-thick sample, for which the behaviour is more complex, for the other $\mathrm{SiO}_{2}$ samples the maximum value of TEEY is attaint for the same energy of primary electrons $(E=540 \mathrm{eV})$. The maximum value of TEEY is much higher than 1, as expected for dielectric materials. It is as high as 4 . Although the local minimum appears at the same energy of primary electrons $(E=1000 \mathrm{eV})$ there is a substantial difference in its depth as function of the $\mathrm{SiO}_{2}$-layer thickness. However, there is no linearity in the local minimum depth as function of the layer thickness. One shall state that for thicknesses up to $25 \mathrm{~nm}$ the reduction of TEEY at the local minimum is of $35 \%$ from the maximum TEEY and for thicknesses above $60 \mathrm{~nm}$ it is as much as $70 \%$. It suggests a threshold dependent behaviour of the value of $\mathrm{Y}_{\min }$ according to the dielectric layer thickness. The rebound of the TEEY after the local minimum is moderate for thicknesses of $9.6 \mathrm{~nm}$ and $25.0 \mathrm{~nm}$, whereas it is more important for $59.7 \mathrm{~nm}$ and $89.9 \mathrm{~nm}$. It might be explained in terms of comparison between the maximum penetration depth of primary electrons and the dielectric layer thickness. The thinner the $\mathrm{SiO}_{2}$ layer is, the less energy is needed for the primary electrons to transit it and to reach the substrate. By comparing the TEEY of $\mathrm{SiO}_{2}$-samples of different thicknesses, between $10 \mathrm{~nm}$ and $90 \mathrm{~nm}$, issued from Monte Carlo based simulation of electrons trajectories in solids made under CASINO software ${ }^{58}$ it appears that the TEEY is increased when the energy of primary electrons, i.e., the maximum penetration depth of primary electrons is similar to the sample thickness. For higher energies of the primary electrons $(E>1500 \mathrm{eV})$ the TEEY becomes independent from the $\mathrm{SiO}_{2}-$ layer thickness, converging toward the same value for all samples (Fig. 8).

As reported in early works on very thin $\mathrm{SiO}_{2}$ layers ${ }^{17,18}$ reasons for the local minimum in the TEEY curve could be the dielectric charging phenomenon due to continuous electron 
irradiation of the dielectric or the dielectric layer thickness in cases for which the latter is larger than the maximum penetration depth of primary electrons. The results obtained here not only confirm the dielectric charging phenomenon as origin of the appearance of a local minimum in the TEEY but go beyond. They show that even though the primary electron beam is pulsed, thus limiting the dielectric charging, the atypical shape of the TEEY curves appears and the local minimum depth depends on the thickness of the dielectric layer. The observed atypical shape of TEEY curves results from a balance between the processes of charging - discharging occurring in the dielectric layer upon electron irradiation.

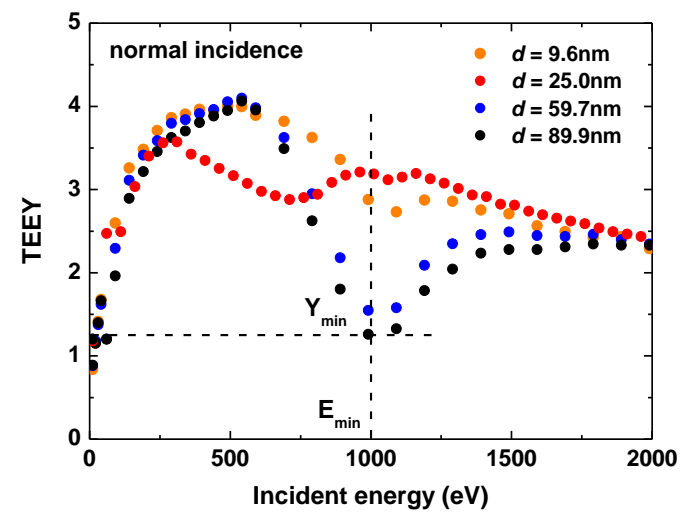

FIG. 8. TEEY of $\mathrm{SiO}_{2}$ thin layers of different thicknesses.

\section{Influence of incident angle of the primary electrons}

To emphasize the effect of the thickness over the TEEY curve, we have performed a study focusing on the incident angle of primary electron beam. The results are shown in Fig. 9 for two $\mathrm{SiO}_{2}$-layers, one very thin $(d=25.0 \mathrm{~nm})$ and one thin layer $(d=89.9 \mathrm{~nm})$, and for three incident angles: normal incidence and incident angles of $45^{\circ}$ and $60^{\circ}$. The atypical shape of the TEEY curve is observed for all incident angles of the primary electron beam. The TEEY curve globally increases with increasing the incidence angle. Indeed, for the very thin layer (Fig. 9a), with a larger incident angle, the primary electrons remain longer near the surface, therefore more secondary electrons are likely to escape, which contributes to the global increase of the TEEY curve. The global increase of the TEEY curve is more noticeable for the very thin layers than for the thin $\mathrm{SiO}_{2}$-layers (Fig. 9). The most probable explanation is a refueling of the holes generated by the secondary electron during their escape due to a recombination induced by penetrating from the substrate electrons in the case of very thin layers. Moreover, the initial slope of TEEY curve, as function of the energy of primary electrons, is steeper for larger incident angles and the maximum TEEY is achieved for higher energies of the primary 
electrons. One also observes a shift of the local minimum of TEEY (Fig. 9a), following the raise of the angle of the incident beam. This inclination increases the distance, and reduces the probability of the primary electrons to reach the substrate. Other interesting feature for the largest incident angle studied here $\left(60^{\circ}\right)$ is that after the local minimum, the TEEY achieves even higher values before starting to slowly decrease for high energies of the primary electrons. The TEEY curve conserves the atypical shape in the case of thin layers but does not show any particular features (Fig. 9b). The maximum achieved TEEY for thin dielectric layers is much smaller, with a factor of 1.5 , compared to that from very thin $\mathrm{SiO}_{2}$-layers.
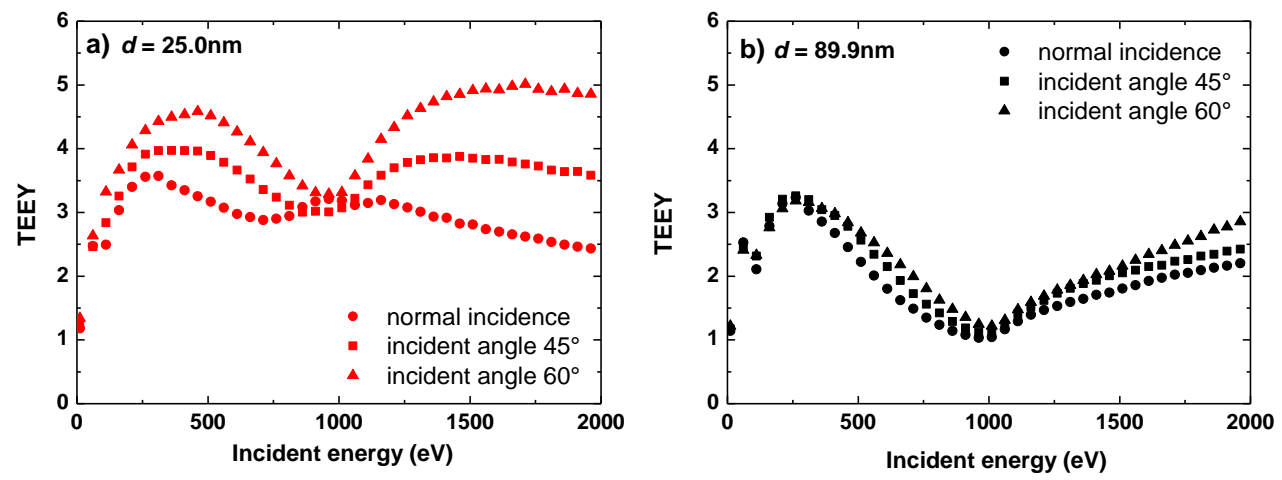

FIG. 9. TEEY curves of (a) very thin $\mathrm{SiO}_{2}$ layer of $25.0 \mathrm{~nm}$ and (b) thin $\mathrm{SiO}_{2}$ layer of $89.9 \mathrm{~nm}$ thickness at different electron beam incident angles.

\section{Application of an external electric field by polarization of the sample holder or of the collector}

Since the electrons are charged particles, applying an external electric field should produce modification of the deposited charges, which in turn will result in modification of the TEEY curve. Two ways of polarization are possible in the employed in this study experimental setup, 1) of the sample holder and 2) of the collector.

The TEEY curves shown in Fig. 10 were obtained for different polarization voltages of the sample holder only. For all polarization voltages of the sample holder, the atypical shape of the TEEY curves is preserved, following the behaviour defined by the thickness of the $\mathrm{SiO}_{2}-$ layers, discussed earlier in Sec. IV.B.1. For layers with thickness $d=9.6 \mathrm{~nm}$ and $59.7 \mathrm{~nm}$, (Figs. 10a and 10b), increasing the applied polarization voltage leads to a global increase of the TEEY curve with a maximum TEEY going up to around 6 for the $9.6 \mathrm{~nm}$-thick layer. However, polarization of the sample holder of $-18 \mathrm{~V}$ is enough to empty out the secondary electrons available in the very thin layers. Beyond that value the TEEY curves become independent of 
the polarization of the sample holder, keeping the same shape for all polarization voltages. Indeed, when increasing the polarization voltage to $-27 \mathrm{~V}$, the TEEY remains the same. The TEEY curves of thin $\mathrm{SiO}_{2}$-layers $(d=89.9 \mathrm{~nm}$, Fig. 10c) do not show any dependence on the polarization voltage of the sample holder. The atypical shape of TEEY, characteristic of thin $\mathrm{SiO}_{2}$-layers and the TEEY-values remain the same.
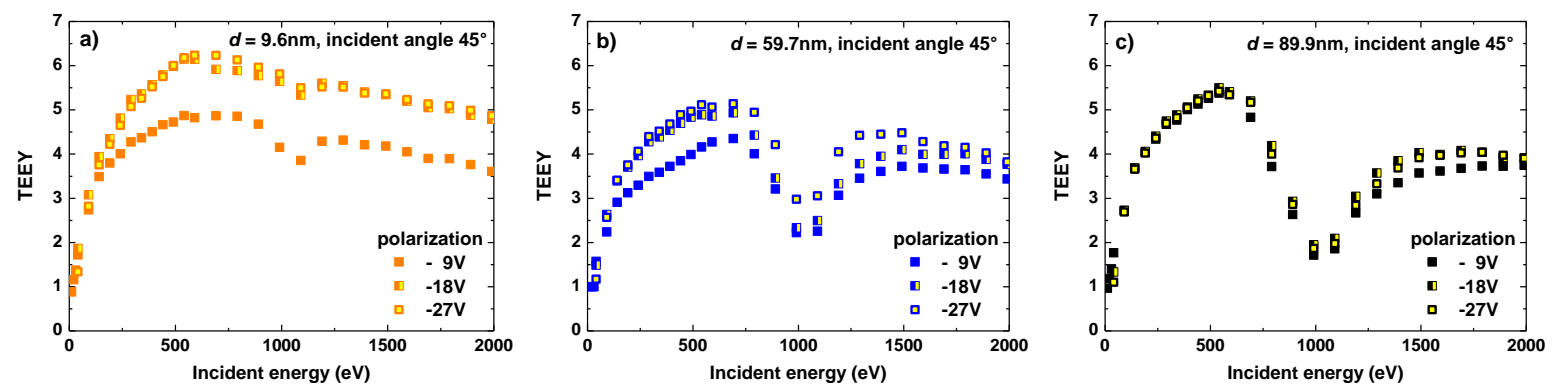

FIG. 10. TEEY of $\mathrm{SiO}_{2}$ layers of thickness (a) $9.6 \mathrm{~nm}$, (b) $59.7 \mathrm{~nm}$ and (c) $89.9 \mathrm{~nm}$ under a $45^{\circ}$ incident electron beam, for different polarization voltages of the sample holder.

It is possible to apply a stronger field by polarizing the collector when studying the effect of an external electric field. The associated TEEY measurements are shown on Fig. 11 for two $\mathrm{SiO}_{2}$-layer thicknesses $(d=25.0 \mathrm{~nm}$ and $89.9 \mathrm{~nm})$. Regardless the dielectric layer thickness, when increasing the electric field by polarization of the collector, the shape of the TEEY curve gets closer to the typical one. This finding is in agreement with the reported results by $\mathrm{Yu}$ et al. ${ }^{17}$ One can suggest that an applied external electric field has a direct impact on the charges and therefore on the TEEY curve, presumably by promoting the secondary electron emission through energetic assistance. For the very thin $\mathrm{SiO}_{2}$-layer $(d=25.0 \mathrm{~nm}$, Fig. 11a), the shape of the TEEY curve reaches the typical one for a polarization of the collector of $V_{\text {coll }}=200 \mathrm{~V}$, in the current geometrical configuration, and remains unchanged for voltages beyond that value. This result can be achieved if all available electrons are extracted from the dielectric layer. For the thin $\mathrm{SiO}_{2}$-layer $(89.9 \mathrm{~nm})$ a much higher polarization should be applied in order to complete the transition to the typical shape of the TEEY curve. It is worth noticing that the non-regular shape of the TEEY curve of the $25.0 \mathrm{~nm}$-thick $\mathrm{SiO}_{2}$-layer without polarization of the collector is not an artefact of the measurement but represents a transition, most likely toward the characteristic properties of bulk materials. Such transition becomes visible at polarizations by at least $V_{\text {coll }}=700 \mathrm{~V}$ for the $89.9 \mathrm{~nm}$-thick $\mathrm{SiO}_{2}$-layer. In addition, the position of the local minimum slightly shifts to higher energies with the raise of externally applied electric field (Fig. 11). This is because a higher electric field assists the charge transport and allows deeper secondary electrons to be extracted. We hereby highlight that the electron 
emission is an internal effect of the dielectric layer under electron irradiation and that the electric field plays a major role on the shape and values of the TEEY curve.
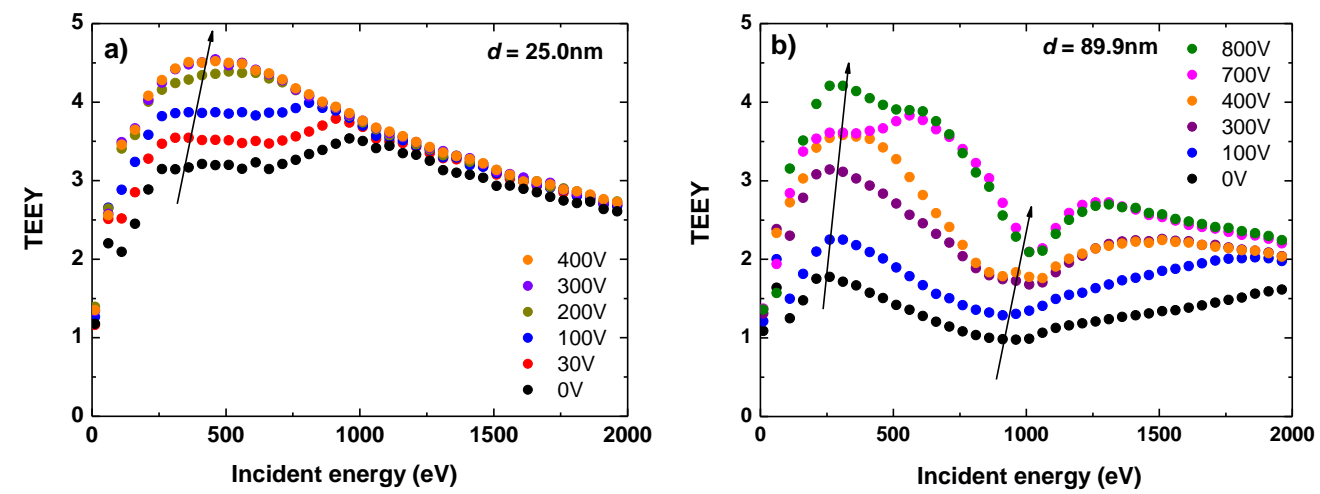

FIG. 11. TEEY curves of (a) very thin $\mathrm{SiO}_{2}$ layer of $25.0 \mathrm{~nm}$ and (b) thin $\mathrm{SiO}_{2}$ layer of $89.9 \mathrm{~nm}$ thickness for different polarization voltages of the collector.

\section{Physical phenomena leading to the atypical shape of the TEEY curve-modeling results}

\section{General trends of the TEEY curve}

Aiming at a better understanding of the physical phenomena at the origin of the atypical shape of the TEEY curve and their relative contributions we have analyzed results from the Dionne's model adapted for thin dielectric layers. Comparison of TEEY curves of a thin $\mathrm{SiO}_{2}$ layer $(d=90 \mathrm{~nm})$ obtained after the Dionne's model is shown in Fig. 12, alongside with the effect of induced space-charge field on the TEEY and of the mechanisms of increased conduction induced by RIC and FDC. From a general point of view, consideration in the Dionne's model (Eq. (8)) of the generation of space-charge electric field as consequence of the dielectric charging after electron irradiation and of the RIC and FDC mechanisms, leads to the atypical shape of the TEEY curve with formation of a local minimum at $1.03 \mathrm{keV}$. The atypical shape of the TEEY curve can be explained by two concomitantly evolving quantities. First, the accumulation of uncompensated positive charges in the dielectric layer during electron emission generates a space-charge electric field. This field is decelerating for the primary electrons and gradually modifies the trajectory of the free internal charges, i.e., the secondary electrons generated in the material. Reduction of the mean free path of the secondary electrons toward the surface, and consequently of the average depth of their escape, strongly reduces the TEEY. Second, the conduction mechanisms, RIC and FDC, lead to a fast discharge of the accumulated, in the dielectric sample, charges through the substrate to the electrical ground. The discharge occurs when the space-charge field exceeds the threshold field for non-linear 
charge transport, so that the FDC mechanism switches on. The conduction in the material becomes thus predominantly governed by the FDC and the resistance of the dielectric layer quickly decreases. The contribution of RIC to the material conduction, although non-negligible, remains limited in the considered here case due to the relatively low doses of primary electrons $(D=20 \mathrm{kGy}$ for $E=1 \mathrm{keV})$. The discharge process reduces the space-charge electric field. As consequence, the TEEY starts increasing again $(E=1.03 \mathrm{keV}$, on Fig. 12) to join the typical TEEY curve.

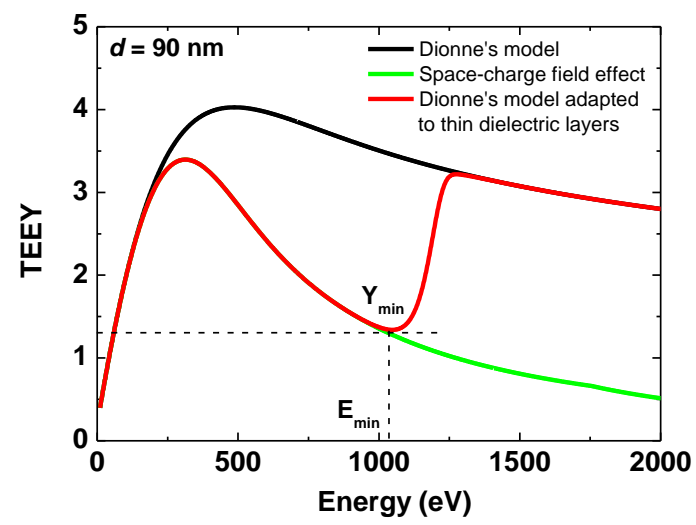

FIG. 12. Simulations of the TEEY of a $90 \mathrm{~nm}$ thick $\mathrm{SiO}_{2}$ thin layer: (i) Typical TEEY curve calculated according the Dionne's model (black curve), (ii) Effect of the space-charge induced electric field on the TEEY (green curve) and (iii) Switch on of the increased material conductivity, due to FDC and RIC mechanisms (red curve).

The Dionne's model adapted for thin dielectric layers that we propose here to describe the atypical TEEY curve reproduces adequately the experimentally observed shape but has several approximations. It includes estimations of the backscattered electron emission yield which is considered constant $(\eta=0.2$, Eq. (9)) but also of the absorption coefficient of the primary electrons at its maximum value $\left(A_{a}\right.$, in Eq. (4)). However, it allows to identify the main mechanisms at the origin of the observed atypical shape of the TEEY curve with the appearance of a well-pronounced dip at energy of the primary electrons of about $1 \mathrm{keV}$, namely the induced space-charge field and the increased material conductivity, including both FDC and RIC mechanisms.

\section{Electric field distribution for a given energy of the primary electrons}

The incident electrons increase the population of both negative and positive charges within the thin $\mathrm{SiO}_{2}$ layer during irradiation. However, the populations of positive and negative charges are localized at different depths in the irradiated dielectric, depending on the energy of incident electrons, and therefore generate an electric field variable with the irradiation history. 
In addition, the competition between charge and discharge in the dielectric sample alters the final electric field distribution. The in-depth profiles of the electric field for different points of interest in the TEEY curve, shown in Fig. 12, are illustrated on Fig. 13.
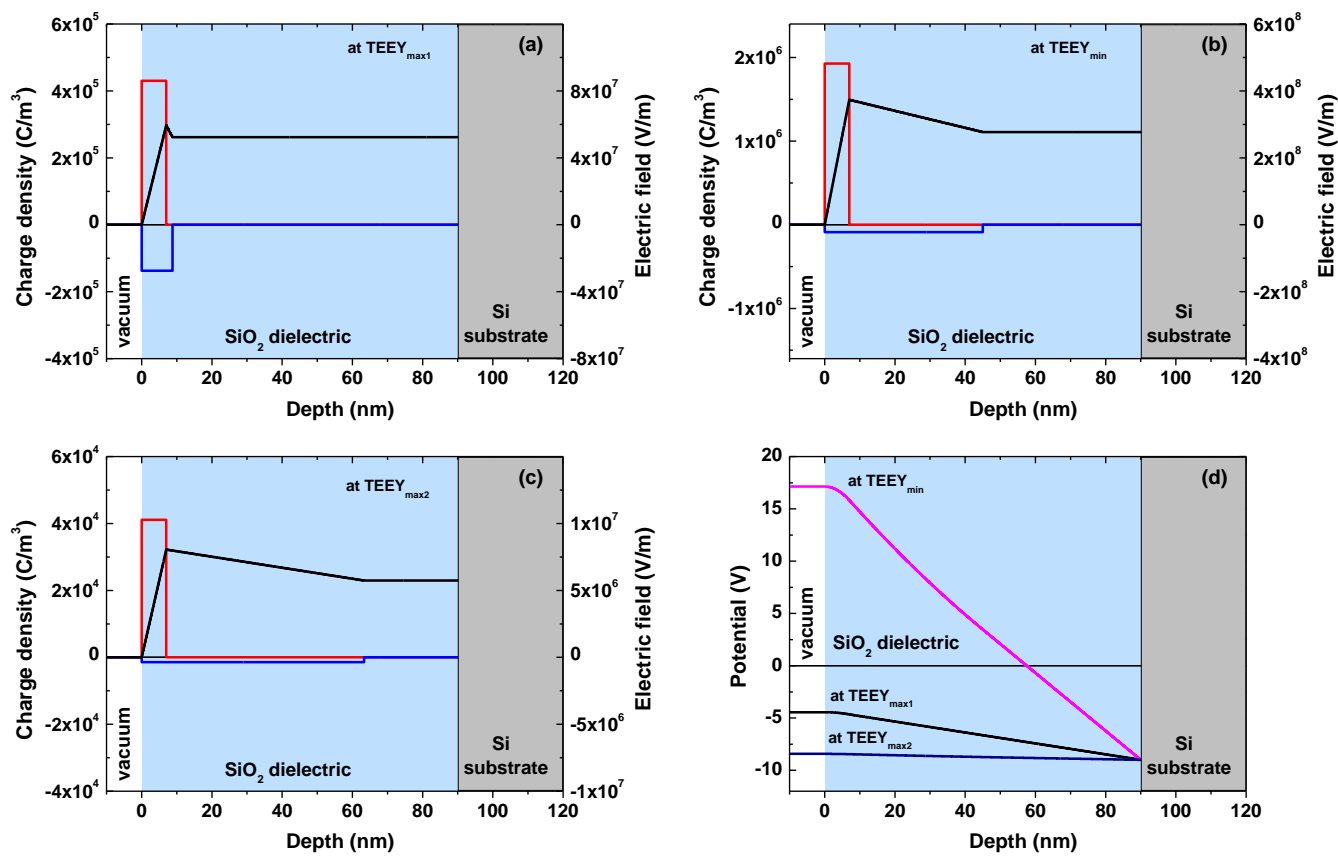

FIG. 13. In-depth distributions of the electric field and the charge densities (positive charges - red curves and negative charges - blue curves) for energies of the primary electrons at specific points of the TEEY curve shown in figure 12: (a) first TEEY maximum, (b) TEEY minimum, $\mathrm{Y}_{\mathrm{m}}$, (c) second TEEY maximum and (d) potential profiles.

The sign of the space-charge electric field is positive when oriented along the $\mathrm{z}$ axis, i.e., in the same direction as the incoming primary electrons, and negative when oriented toward the vacuum. The electric field in the dielectric bulk is maximum at $\lambda_{\mathrm{e} 0}=7.0 \mathrm{~nm}$, corresponding to the limit escape depth of secondary electrons. The magnitude of the space-charge electric field firstly increases with the incident energy (Fig. 13a and 13b), then decreases (Fig. 13c) when the material conductivity associated with FDC and RIC mechanisms causes a progressive discharge of the thin $\mathrm{SiO}_{2}$ layer.

The very low values of electric field in the vacuum and near the dielectric layer surface (of only 90, -340 and 170 V/m for cases (a), (b) and (c), respectively), are due to the large distance $(w=10 \mathrm{~cm})$ that separates the sample surface to the ground (Fig. 3b). A negative value (field reversal) is observed only for the case corresponding to the TEEY minimum. Such field reversal situation is often reported in the literature, notably for the case of metal-coated surfaces. ${ }^{43,59}$ Occurrence and in-depth position of the field inversion are dependent on various factors: potential applied to the substrate, position of the top electrode, and density, and position 
of stored charges. The only case of field inversion occurs here for electron energy corresponding to the minimum of TEEY $\left(\mathrm{Y}_{\mathrm{min}}\right)$ and maximum of positive charges density. The position of the field inversion is very close to the surface $(<<0.1 \mathrm{~nm})$. This behavior results from both the large distance of the ground from the sample surface $(w=10 \mathrm{~cm})$ compared to the sample thickness $(d=90 \mathrm{~nm})$ and the moderate charging expected using pulsed irradiation conditions. Consideration of the potential profiles of Fig. 13d confirms this 'moderate' charging: the potential variation introduced by space-charge effects remains smaller than the applied potential except for the case of $Y_{\min }$. Note here that the potential varies linearly from the sample surface to the ground situated at $10 \mathrm{~cm}$ away to the left (Fig. 13).

\section{Competition between dielectric charging and discharging phenomena along electron irradiation}

The electron irradiation increases the space-charge field, which reduces the mean escape depth of secondary electrons and consequently influences the TEEY curve. From the layered structure approximation, the calculated space-charge field is introduced into the Fitting's equation to calculate the mean escape depth of secondary electrons (Eq. (9)). The simulated values of the maximum space-charge electric field and mean escape depth of secondary electrons $\left(\lambda_{\mathrm{e}}\right)$ defined by the penetration depth of primary electrons $\left(P_{r}\right)$ as function of the energy of primary electrons during electron irradiation are presented on Fig. 14.

Increasing the space-charge field reduces the mean escape depth of secondary electrons, which means that less generated secondary electrons are able to reach the surface and get emitted from the sample. This decrease of $\lambda_{e}$ therefore reduces the value of TEEY. The charge accumulation in the $\mathrm{SiO}_{2}$-layer creates a space-charge field that might be sufficiently high to halve the mean escape depth of secondary electrons (Fig. 14b).
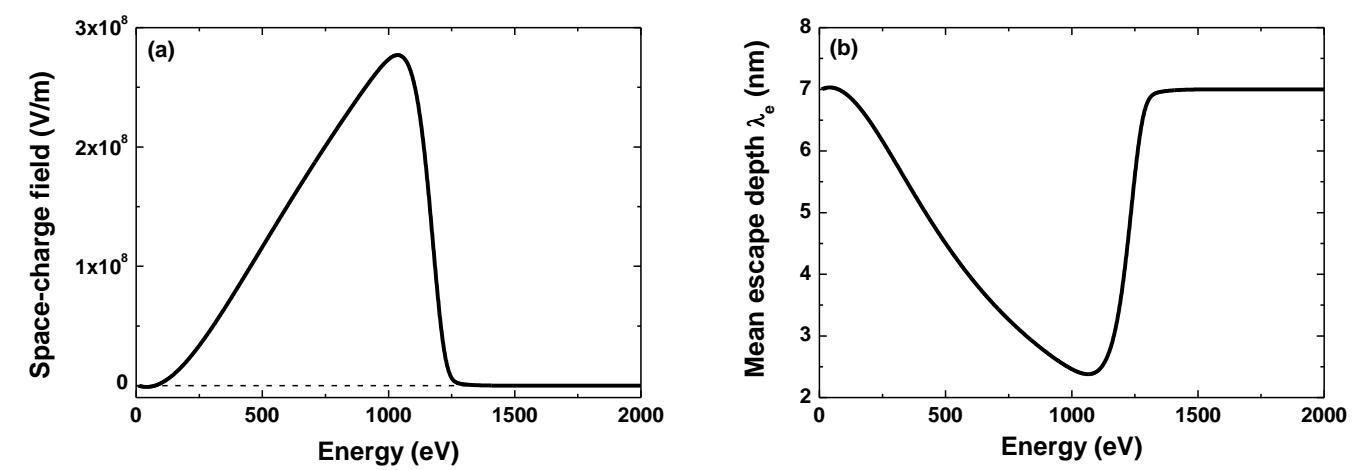

FIG. 14. (a) Space-charge electric field and (b) mean escape depth of secondary electrons as function of the energy of primary electrons during electron irradiation. 
The non-linear process of FDC switched on by the threshold electric field required for $\mathrm{SiO}_{2}$ and reinforced by the RIC mechanism, outweighs the charge buildup in the dielectric layer and thus marks the limitation of the space-charge field effect on the TEEY curve. It is found to appear at energy of the primary electrons of $1.03 \mathrm{keV}$. As a result, the mean escape depth of secondary electrons starts to increase so does the magnitude of TEEY, to finally reach the typical TEEY-values for high energies of the primary electrons.

As already commented the evacuation of the trapped electrical charges is favored by the FDC mechanism, with a small input from the RIC process. In fact, the RIC process brings only a weak contribution to the anomalous TEEY behavior compared to the FDC process. The most likely reason is that the deposited dose is rather mild $(D=20 \mathrm{kGy}$ for incident electron energy of $1 \mathrm{keV}$ ) while relatively high fields can be reached (nearly $3 \times 10^{8} \mathrm{~V} / \mathrm{m}$ according to Fig. 14a). On Fig. 15 is plotted the resistance of the irradiated part of the $\mathrm{SiO}_{2}$-layer, noted $R_{1,2}$, the non-irradiated part of the $\mathrm{SiO}_{2}$-layer, noted $R_{3}$ and the resistance of the equivalent circuit, noted $R_{e q}$, as a function of the energy of primary electrons (equivalent circuit shown in Fig. $3 \mathrm{c}$ ). $R_{1,2}$ and $R_{3}$ are calculated from Eqs. (19a, 19b, 19c), considering the corresponding maximum penetration depth of primary electrons $\left(P_{r}\right)$, for each energy of primary electrons, and the irradiated area $(S)$. The maximum penetration depth of primary electrons $\left(P_{r}\right)$ increases with the incident energy, so does the value of $R_{1,2}$ until the switch on of the FDC. This increase of $R_{1,2}$ is also due to a decrease in irradiation dose upon increase of the irradiation energy. Since less and less dielectric part remains non-irradiated, $R_{3}$ decreases. All considered, the equivalent resistance decreases. When the induced space-charge electric field exceeds the threshold field for FDC which appears at energy of the primary electrons of $750 \mathrm{eV}, R_{3}$, so does the equivalent resistance, starts to decrease significantly. The resistance $R_{1,2}$ achieves its maximum for a bit higher energy of the primary electrons, at $E=820 \mathrm{eV}$, compared to the energy for FDC. One can consider that this delay gives an estimation of the RIC input to the discharge process. At the crossing point of the two resistances $(E=1.03 \mathrm{keV})$ the equivalent resistance of the $\mathrm{SiO}_{2}-$ layer is significantly lower than the initial resistance, i.e., the one of a non-irradiated $\mathrm{SiO}_{2}$-layer, in this case 7 orders of magnitude. The $R_{1,2}$ involving FDC and RIC mechanisms becomes low enough to trigger a discharge of the sample leading to an increase of TEEY. The space-charge induced electric field decreases to zero and the TEEY defined by the model adapted to thin dielectric layers reaches the regular TEEY value, as obtained from the Dionne's model. At an energy of primary electrons of $1.75 \mathrm{keV}$ and higher, the maximum penetration depth of primary 
electrons becomes equal to the $\mathrm{SiO}_{2}$ thin layer thickness $\left(R_{3}=0\right)$ and then conduction mechanisms, induced by irradiation, totally dominate and determine the equivalent resistance.

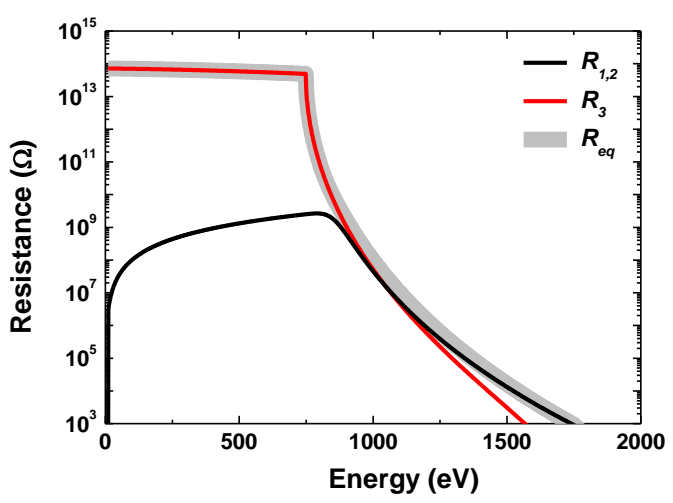

FIG. 15. Resistance extracted from the model: the resistance of irradiated $\left(R_{1,2}\right)$ and non-irradiated $\left(R_{3}\right)$ parts of a $90 \mathrm{~nm}$-thick $\mathrm{SiO}_{2}$-layer, and the resistance of the equivalent circuit $\left(R_{e q}\right)$.

\section{Comparison between experimental and modeled TEEY curves}

Figure 16 shows a comparison between the modeled TEEY curve for a $\mathrm{SiO}_{2}$ layer $(d=90.0 \mathrm{~nm})$ and an experimentally obtained one for $\mathrm{SiO}_{2}$-layer $(d=90.3 \mathrm{~nm})$. One can state that taking into account the space-charge electric field as well as the FDC and RIC mechanisms in the Dionne's model adapted to thin dielectric is sufficient to faithfully reproduce the atypical shape of TEEY curves of thin dielectric layers both qualitatively and quantitatively. Comparison between the modeled and the measured TEEY curves shows that at low energies of primary electrons the slope of the modeled TEEY curve is less steep than the experimental one. This discrepancy most likely stems to originate from the considered constant BEEY in the model. An energy dependent relation for the BEEY would improve the fit for energies of the primary electrons up to $100 \mathrm{eV}$. The maximum of the TEEY curve appears for a bit higher energy of primary electrons $(E=325 \mathrm{eV})$ compared to the measured one. The maximum values (first maximum of TEEY) of the modeled and experimentally measured TEEY are identical. This only confirms that the model considers the main mechanisms at play. It should be recalled here that the proposed model is 1D with coarse treatment of dose and charge profiles. The local minimum $\mathrm{Y}_{\min }$ of the modeled TEEY is less deep than the one obtained experimentally, suggesting a stronger space-charge electric field in reality. Additional physical phenomena like lowering of the Schottky barrier at the interface material/vacuum and the electrons/holes recombination ${ }^{43}$ might improve the comparison. The position of $Y_{\min }$ however, is in fair well agreement. Although simple, the Dionne's model adapted to dielectrics that we propose here is 
very good agreement with the experimentally obtained TEEY curves, able to explain the atypical shape of TEEY curves of thin dielectrics layers.

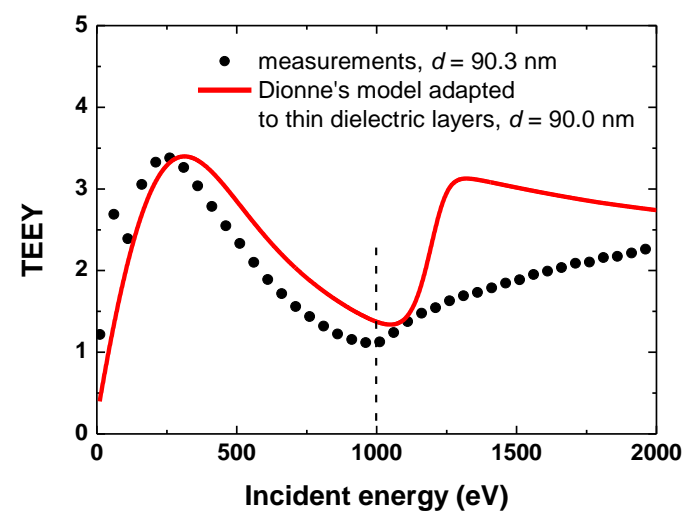

FIG. 16. Comparison of TEEY curves obtained from the Dionne's model adapted for thin dielectrics and from the experiment for a $\mathrm{SiO}_{2}$ layer of $90 \mathrm{~nm}$ thickness.

\section{CONCLUSION}

The main objective of this work is to experimentally determine the electron emission yield from $\mathrm{SiO}_{2}$ layers of thickness below $100 \mathrm{~nm}$. To that end thermally grown $\mathrm{SiO}_{2}$ samples are studied. The TEEY curves are measured for low energies of the primary electrons, in the range $[0,2000 \mathrm{eV}]$. For all studied samples an atypical shape of the TEEY curve exhibiting a local minimum has been found. To confirm the inherent nature of the recorded atypical shape of the TEEY curve of thin dielectric layers, several precautions have been undertaken during the experiments: $(i)$ the thickness of each $\mathrm{SiO}_{2}$ layer is precisely measured, including a mapping procedure having contribution to the performed analysis of possible inhomogeneity-induced effects related to the thickness of the dielectric layers, (ii) the structural properties of the studied $\mathrm{SiO}_{2}$ dielectric layers are studied and defined for each sample, (iii) the surface contamination has been measured just before the TEEY measurements, (iv) the primary electrons are sent by bunches of short pulses to limit the dielectric charging phenomenon, and (v) the energy of primary electrons is increased by steps large enough to prevent residual dielectric charging from previous electron pulses. The observed atypical shape of the TEEY curves is found to depend on the thickness of the $\mathrm{SiO}_{2}$-layers. The TEEY local minimum is more pronounced for samples with thicknesses larger than $25 \mathrm{~nm}$, scaling down to approximately TEEY $\approx 1$. The atypical shape of the TEEY curves is maintained also when applying an external electric field. To get inside the observed phenomenon a 1D-model of TEEY, based on the well-known Dionne's model but adapted to thin dielectric layers, has been developed. The model reveals that the 
atypical shape of TEEY from thin dielectric layers is due to a space-charge electric field induced by the remaining non-compensated positive electric charge in the region, just beneath the sample surface where the secondary electrons are originated, and the irradiated by primary electrons part of the sample. The local TEEY minimum appears because of the created spacecharge electric field that substantially reduces the mean escape depth of secondary electrons. When the space-charge electric field becomes larger than the one inducing non-linear charge transport, the FDC mechanism switches on, and compensates the accumulated in the dielectric layer charges by electrical discharges through the substrate. When the conducting path opens and the sample gets partially discharged, a lowering of the space-charge electric field is observed, resulting in an increase of the mean escape depth of secondary electrons, and therefore of the TEEY. For higher energies of the primary electrons, the RIC mechanism strengthens the FDC one. The contribution of RIC to the material conduction, although nonnegligible, remains limited due to the relatively low doses of primary electrons used in the performed experiments.

This atypical shape of the TEEY curve and the studied physical phenomena can be applied to prevent electrostatic discharges appearing in neighbor dielectric parts of devices for space applications. Depending on the nature of the dielectric material, for example, for a given dielectric layer, a tuning of its thickness would lead to setting a specific electron emission yield under irradiation, and therefore allow a strict control of the dielectric charging phenomenon at the required energy of primary electrons.

\section{ACKNOWLEDGMENT}

Research supported by the program IDEX Actions Thématiques Stratégiques - ATS 2015 of the Université de Toulouse under project SEPHIR (2016-066-CIF-D-DRVD).

\section{DATA AVAILABILITY}

The data that support the findings of this study are available from the corresponding author upon reasonable request. 


\section{REFERENCES}

${ }^{1}$ H. Bruining, Physics and Applications of Secondary Electron Emission (McGraw-Hill Book Co., Inc., New York, 1954).

${ }^{2}$ I.A. Glavatskikh, V.S. Kortov, and H.-J. Fitting, J. Appl. Phys. 89, 440 (2001).

${ }^{3}$ Y. Lin and D.C. Joy, Surf. Interface Anal. 37, 895 (2005).

${ }^{4}$ H. Seiler, J. Appl. Phys. 54, R1 (1983).

${ }^{5}$ B.L. Thiel and M. Toth, J. Appl. Phys. 97, 051101 (2005).

${ }^{6}$ J. Hren, Introduction to Analytical Electron Microscopy. (Springer, New York, NY, 2013).

${ }^{7}$ I.P. Raǐzer, Gas Discharge Physics (Springer, Berlin ; New York, 1997).

${ }^{8}$ A. Dunaevsky, Y. Raitses, and N.J. Fisch, Phys. Plasmas 10, 2574 (2003).

${ }^{9}$ N. Balcon, D. Payan, M. Belhaj, T. Tondu, and V. Inguimbert, IEEE Trans. Plasma Sci. 40, 282 (2012).

${ }^{10}$ K. Ohmi, Phys. Rev. Lett. 75, 1526 (1995).

${ }^{11}$ K. Yokoo, J. Vac. Sci. Technol. B Microelectron. Nanometer Struct. 12, 801 (1994).

12 J.-M. Torres and R.S. Dhariwal, Nanotechnology 10, 102 (1999).

${ }^{13}$ K. Makasheva, G.J.M. Hagelaar, J.-P. Boeuf, T. Callegari, and L.C. Pitchford, IEEE Trans. Plasma Sci. 36, 1236 (2008).

14 T. Iwamatsu, A. Tsutsui, and H. Yamaji, Appl. Phys. Lett. 114, 053511 (2019).

${ }^{15}$ R.B. Horne, M.W. Phillips, S.A. Glauert, N.P. Meredith, A.D.P. Hands, K.A. Ryden, and W. Li, Space Weather 16, 1202 (2018).

${ }^{16}$ J.-C. Mateo-Velez, T. Paulmier, A. Sicard, B. Dirassen, and D. Payan, IEEE Trans. Plasma Sci. 47, 3885 (2019).

${ }^{17}$ S. Yu, T. Jeong, W. Yi, J. Lee, S. Jin, J. Heo, J.M. Kim, and D. Jeon, Appl. Phys. Lett. 79, 3281 (2001).

${ }^{18}$ W. Yi, T. Jeong, S. Yu, J. Lee, S. Jin, J. Heo, and J.M. Kim, Thin Solid Films 397, 170 (2001).

${ }^{19}$ R. Hoffmann and J.R. Dennison, IEEE Trans. Plasma Sci. 40, 298 (2012).

${ }^{20}$ G.F. Dionne, J. Appl. Phys. 44, 5361 (1973).

${ }^{21}$ A. Melchinger and S. Hofmann, J. Appl. Phys. 78, 6224 (1995).

22 J. Cazaux, J. Appl. Phys. 85, 1137 (1999).

${ }^{23}$ H.-J. Fitting, E. Schreiber, J.-Ch. Kuhr, and A. von Czarnowski, J. Electron Spectrosc. Relat. Phenom. 119, 35 (2001).

${ }^{24}$ J. Cazaux, J. Appl. Phys. 95, 731 (2004).

25 J. Cazaux, Scanning 26, 181 (2006).

${ }^{26}$ H.-J. Fitting, M. Touzin, and E. Schreiber, Phys. Status Solidi C 8, 1282 (2011).

${ }^{27}$ D.A.G. Bruggeman, Ann. Phys. 416, 636 (1935).

${ }^{28}$ SOPRA S.A., WinElli Software, Version 3.04 (1999). 
${ }^{29}$ T. Gineste, M. Belhaj, G. Teyssedre, and J. Puech, Appl. Surf. Sci. 359, 398 (2015).

${ }^{30}$ C. Rigoudy, K. Makasheva, G. Teyssedre, L. Boudou, M. Belhaj, and S. Dadouch, in 2018 IEEE 2nd Int. Conf. Dielectr. ICD (IEEE, Budapest, 2018), pp. 1-4 (DOI: 10.1109/ICD.2018.8514648).

${ }^{31}$ H.-B. Zhang, X.-C. Hu, R. Wang, M. Cao, N. Zhang, and W.-Z. Cui, Rev. Sci. Instrum. 83, 066105 (2012).

${ }^{32}$ G.F. Dionne, J. Appl. Phys. 46, 3347 (1975).

${ }^{33}$ H.-J. Fitting, Phys. Status Solidi A 26, 525 (1974).

${ }^{34}$ H. Bethe, Ann. Phys. 397, 325 (1930).

${ }^{35}$ H.T. Nguyen-Truong, Ultramicroscopy 149, 26 (2015).

${ }^{36}$ R.G. Lye and A.J. Dekker, Phys. Rev. 107, 977 (1957).

${ }^{37}$ H.-J. Fitting, H. Glaefeke, and W. Wild, Phys. Status Solidi A 43, 185 (1977).

${ }^{38}$ J. Cazaux, Appl. Surf. Sci. 257, 1002 (2010).

${ }^{39}$ X. Meyza, D. Goeuriot, C. Guerret-Piécourt, D. Tréheux, and H.-J. Fitting, J. Appl. Phys. 94, 5384 (2003).

${ }^{40}$ H.-J. Fitting and J. Boyde, Phys. Status Solidi A 75, 137 (1983).

${ }^{41}$ J.P. Ganachaud and A. Mokrani, Surf. Sci. 334, 329 (1995).

${ }^{42}$ M. Belhaj, T. Tondu, and V. Inguimbert, J. Phys. Appl. Phys. 42, 145306 (2009).

${ }^{43}$ M. Touzin, D. Goeuriot, C. Guerret-Piécourt, D. Juvé, D. Tréheux, and H.-J. Fitting, J. Appl. Phys. 99, 114110 (2006).

${ }^{44}$ A. Pugliara, C. Bonafos, R. Carles, B. Despax, and K. Makasheva, Mater. Res. Express 2, 065005 (2015).

${ }^{45}$ A. Pugliara, M. Bayle, C. Bonafos, R. Carles, M. Respaud, and K. Makasheva, Mater. Res. Express 5, 035027 (2018).

${ }^{46}$ J.F. Fowler, Proc. R. Soc. Lond. Ser. Math. Phys. Sci. 236, 464 (1956).

${ }^{47}$ T. Paulmier, B. Dirassen, M. Belhaj, and D. Rodgers, IEEE Trans. Plasma Sci. 43, 2894 (2015).

${ }^{48}$ K.-C. Kao and W. Hwang, Electrical Transport in Solids: With Particular Reference to Organic Semiconductors, 1st ed (Pergamon Press, Oxford; New York, 1981).

${ }^{49}$ C. Rigoudy, K. Makasheva, M. Belhaj, S. Dadouch, G. Teyssedre, and L. Boudou, in 2020 IEEE 3rd Int. Conf. Dielectr. ICD (IEEE, Valencia, Spain, 2020), pp. 205-208.

${ }^{50}$ C. Villeneuve-Faure, K. Makasheva, L. Boudou, and G. Teyssedre, Nanotechnology 27, 245702 (2016).

${ }^{51}$ J. Robertson, Eur. Phys. J. Appl. Phys. 28, 265 (2004).

${ }^{52}$ K. Makasheva, B. Despax, L. Boudou, and G. Teyssedre, IEEE Trans. Dielectr. Electr. Insul. 19, 1195 (2012).

${ }^{53}$ The MathWorks, Inc., MatLab R2018b (2018).

${ }^{54}$ W.M. Haynes ed., CRC Handb. Chem. Phys., CRC Press (Boca Raton, Fl, 2011).

${ }^{55}$ A.G. Xie, S.R. Xiao, and H.Y. Wu, Indian J. Phys. 87, 1093 (2013). 
${ }^{56}$ C.T. Kirk, Phys. Rev. B 38, 1255 (1988).

${ }^{57}$ R.A.B. Devine, J.-P. Duraud, and E. Dooryhée, editors, Structure and Imperfections in Amorphous and Crystalline $\mathrm{SiO}_{2}$ (Wiley, Chichester; New York, 2000).

${ }^{58}$ D. Drouin, A.R. Couture, D. Joly, X. Tastet, V. Aimez, and R. Gauvin, Scanning 29, 92 (2007).

${ }^{59}$ J. Cazaux, J. Appl. Phys. 59, 1418 (1986). 